\title{
Calculation of atmospheric neutrino flux
}

\author{
V.Plyaskin \\ Institute of Theoretical and Experimental Physics, \\ Moscow, Russia
}

March 21, 2001.

\begin{abstract}
A calculation of the fluxes of primary particles arriving to the Earth's vicinity as well as those produced in the interactions of the primaries with the atmosphere is presented. The result of calculations is compared with the experimental data obtained with the Alpha Magnetic Spectrometer (AMS). A good agreement of calculated and measured fluxes of charged particles supports the viability of the atmospheric neutrino flux calculation.
\end{abstract}

\section{Introduction}

The interpretation of the data on the atmospheric neutrinos obtained by different experiments [1, 2, 3, 4, 5] strongly relies on the calculations of neutrino fluxes [6, 7, 8, 9, 10, 11, 12, 13, 14, 15. Starting from a common assumption that the primary cosmic rays arrive in the vicinity of the Earth isotropically the calculations differ in the level of sophistication used by different authors to describe the production of the secondaries in the hadronic interactions of primary cosmic particles (mostly protons) with the atmosphere and in the way the secondaries are propagated in the Earth's magnetic field. Different calculations producing considerably different results, the validity of neutrino flux calculations can only be checked by comparing the predictions of these calculations with the experimental data. Although the difference in the measured and calculated atmospheric fluxes of upgoing and downgoing neutrinos is considered as a clear evidence for neutrino oscillations [15] it is nevertheless recognized [20], that the same calculations [7, 17] seem to fail to properly describe the measured east-west asymmetry of atmospheric neutrino fluxes [16] related to a well known [18, 19] observation that due to the influence of the Earth's (nearly dipole) magnetic field the fluxes of the primary predominantly positive (p, $\alpha$-particles) hadronically interacting cosmic rays reaching the atmosphere contain more particles coming from the west than from the east. Thus, more accurate and detailed calculations describing propagation of primary cosmic rays and their interactions with the atmosphere is needed. 
The recent data from the Alpha Magnetic Spectrometer (AMS) 23, 24, 25] provide a vast amount of information on the fluxes of both primary and secondary particles detected in the near Earth orbit covering most of the Earth's surface. The AMS data display not only the common features of the magnetic cutoff dependence on the distance from the magnetic equator but also reveal the presence of particles well under the cutoff [24, 25]. These particles originate from the production and eventual decay of the secondaries produced in the inelastic interactions of the primary cosmic particles with the atmosphere and consequently carry the information about neutrinos produced in decays of the secondaries. A comparison of the results obtained in the calculations with the fluxes of the secondaries measured with AMS represents a means to prove or disprove the validity of the results of the calculations concerning the predictions of atmospheric neutrino fluxes.

In the following the result of calculation of the near Earth fluxes of charged particles and atmospheric neutrinos is presented. First, the model used and its parameters is described. Section 3 describes the origin of the particles near the Earth. Section 4 is devoted to results of calculations of fluxes of charged particles at the altitude of $400 \mathrm{~km}$ from the Earth's surface corresponding to the AMS flight altitude. The simulated fluxes of charged particles are compared with those measured with AMS in section 5. Section 6 is devoted to the results of calculations of atmospheric neutrino fluxes at the ground level in general. The neutrino fluxes at different experimental sites are discussed in section 7 . The last section gives a summary of the results and draws some conclusions.

\section{Description of the model}

The calculation uses the Monte Carlo technique and is based on the GEANT3/GHEISHA package [26]. This package is extensively used for the simulation of processes in various detectors of high energy physics experiments. To use this package for the simulation on a scale of propagation of cosmic rays in the Earth's magnetic field, their interactions with the Earth's atmosphere and the Earth itself, followed by production, propagation and decay of secondary particles, the parameters of the corresponding media are adapted to scale them from a thousands of kilometer scale of cosmic ray travel and interaction to a centimeter scale characteristic for GEANT based simulations.

The Earth is modeled to be a sphere of $6378.14 \mathrm{~km}$ radius of a uniform density of 5.22

$\mathrm{g} / \mathrm{cm}^{3}$ derived from the total mass of the Earth. The atomic and nuclear properties of the Earth are taken to be those of Ge - the closest in density $\left(5.32 \mathrm{~g} / \mathrm{cm}^{3}\right)$ element. The Earth's atmosphere is modeled by $1 \mathrm{~km}$ thick variable density layers of air extending up to $71 \mathrm{~km}$ from the Earth's surface. The density change with altitude is calculated using a Standard Atmosphere Calculator [27].

The Earth's magnetic field for the year 1998 is calculated according to World Magnetic Field Model (WMM-2000) [28] with 6 degrees of spherical harmonics. The WMM-2000 is a predictive model based on the magnetic field measurements on a global scale. 
The flux $(\Phi)$ of high energy primary protons in the Solar system is parametrized on the basis of the existing experimental data [24, 29, 30, 31, 32, 33. by a power low in rigidity $(\mathrm{R}), \Phi(R) \sim R^{-\gamma}$ with $\gamma=2.79$. At the energies below several $\mathrm{GeV}$ the spectrum is corrected for the solar activity to be similar to that of the year 1976 34 - the year of a similar solar activity as in the year 1998 according to the 11 year solar cycle. Primary protons are isotropically emitted from a sphere of 10 Earth's radii. The long distance from the Earth of the emission point is sufficient for a particle to endure sufficient influence of the Earth's magnetic field to form an adequate topology of the flux in the Earth's vicinity.

Secondary particles produced in the interactions with the atmosphere and the Earth are traced in the magnetic field following a normal GEANT interaction/decay procedure.

\section{Origin of particles near Earth}

The shape of magnetic field determines the behaviour of charged particles at their approach to the Earth and the eventual motion of primaries and secondaries in the Earth's vicinity. The channeling of the particles in the Earth's magnetic field leads to a well known effect that the flux of particles coming from space is at its maximum at the magnetic poles (see top Fig (1). This effect is associated with the magnetic cutoff of low momenta particles in the equator region. A peculiar feature of particle channeling from the outer space is that most of the particles detected in the pole region in the Earth's vicinity have their origin in the equator region of the isotropically shining sphere at 10 Earth's radii used in the simulation (bottom Fig (1). The efficiency of channeling of the particles depends on the emission parameters such as particle momentum and emission direction.

Figure 2 shows the emission parameters of the particles reaching the Earth. One can see in figure 2 that small momentum particles even emitted in the direction opposite to the Earth can reach the Earth's vicinity whereas high momentum particles can do that only when the initial direction is close to a straight line between the emission point and the Earth. The dashed lines in figure 2 show the cuts on the emission parameters used in the simulation. An additional cut is set on the momentum of primary protons. It is set to be of $0.5 \mathrm{GeV} / \mathrm{c}$ for two reasons. First, due to the detector material thickness the minimum kinetic energy required for a proton to trigger AMS is $\sim 0.13 \mathrm{GeV}$ corresponding to a proton momentum of $0.5 \mathrm{GeV} / \mathrm{c}$. Secondly, the kinetic energy threshold of charged pion production in p-nucleus collisions is $\sim 0.16 \mathrm{GeV}$ 35] corresponding to a proton momentum of $0.54 \mathrm{GeV} / \mathrm{c}$. Protons with energies below the threshold can not produce charged pions which represent the source of detectable secondaries and eventually of atmospheric neutrinos. The cut on the proton momentum eliminates the few events in the region forbidden by the cut of the bottom Fig 2 
Of all protons with the momentum spectrum described above uniformly emitted from the sphere of 10 Earth's radii only a $7.4 * 10^{-4}$ part reaches the Earth's atmosphere. In the subsequent simulation only particles with initial kinematics compatible with those reaching the Earth's atmosphere are traced the whole way until they interact with the atmosphere or the Earth itself or/and continue the journey beyond a sphere of 15 Earth's radii. The charged secondaries from the interactions are traced until they go below a limit of $0.125 \mathrm{GeV} / \mathrm{c}$.

Figure 3] shows an example of the trajectory of a particle reaching the Earth. (Note that the AMS flight altitude of $400 \mathrm{~km}$ corresponds to 1.06 Earth's radii). Due to influence of the Earth's magnetic field the direction of a particle at its entrance to the atmosphere is by far different from the initial direction.

Primary cosmic particles may endure an elastic or inelastic scattering in the atmosphere thus changing their direction and/or momentum. Figure 4 shows the trajectory of such a particle which eventually reenter the atmosphere but at a place far away from the point of initial interaction. As it will be shown later, there is a considerable amount of such particles in the total flux of incoming cosmic rays.

Secondary particles produced in the inelastic interactions of primary cosmic rays with the atmosphere follow a usual pattern of hadronic or electromagnetic cascade and most of them quickly end up in the atmosphere itself or in the Earth. However, under the influence of the Earth's magnetic field some (predominantly stable) particles from the atmospheric showers escape upward from the atmosphere and travel long distances before being absorbed in the atmosphere. The trajectory of such particle depends on the kinematics of the particle production and the geomagnetic coordinates of the origin. Unless they interact with the atmosphere the particles with the same rigidity $(\mathrm{p} / \mathrm{z})$ follow the same trajectory. Figure 5 shows the trajectory of a positive particle produced in the atmosphere in the northern hemisphere, reflected at a mirror point in the southern hemisphere before being absorbed at an approximatively the same latitude but a different longitude.

The spiraling of the particles, their motion from and to the Earth's surface as well as their bouncing across and drifting along the magnetic equator is determined by the shape of the Earth's magnetic field. Depending on the production place and direction with respect to the Earth's surface secondary particles can make a return trip as illustrated in Fig [5 a one way trip across the magnetic equator, a multiple bounce (Fig [6) or even a round the globe trip (Fig 7). At the limit a secondary particle produced in the atmosphere over a certain limited region of the Earth (called South Atlantic Anomaly) can have a closed trajectory (Fig 8) and could live forever if it would not interact with the atmosphere at the place where it was created nor lose its energy on synchrotron radiation.

As one can see in figures $5 \mathrm{~d}, 6 \mathrm{~d}, 7 \mathrm{~d}$ and $8 \mathrm{~d}$ the particles in their motion pass the level of the AMS flight (1.06 Earth's radii) going up and down several times. The geographic coordinates of the place over which a secondary particle can be detected at a certain altitude is connected with the place of origin of the particle. 

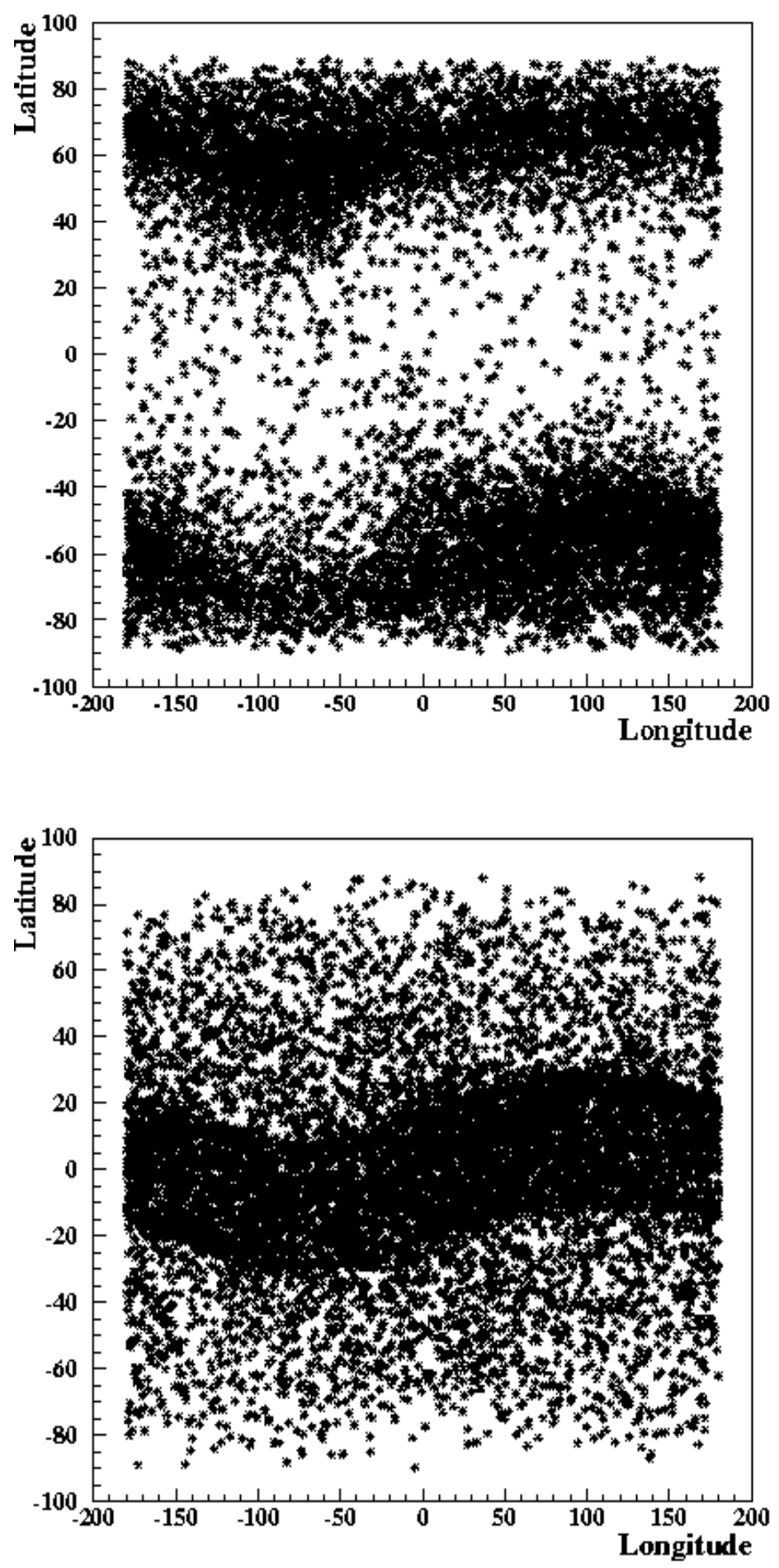

Figure 1: Top: The points of impact of primary cosmic rays on the Earth. Bottom: The points of origin on the 10 Earth's radii sphere of primary cosmic rays reaching the Earth. 

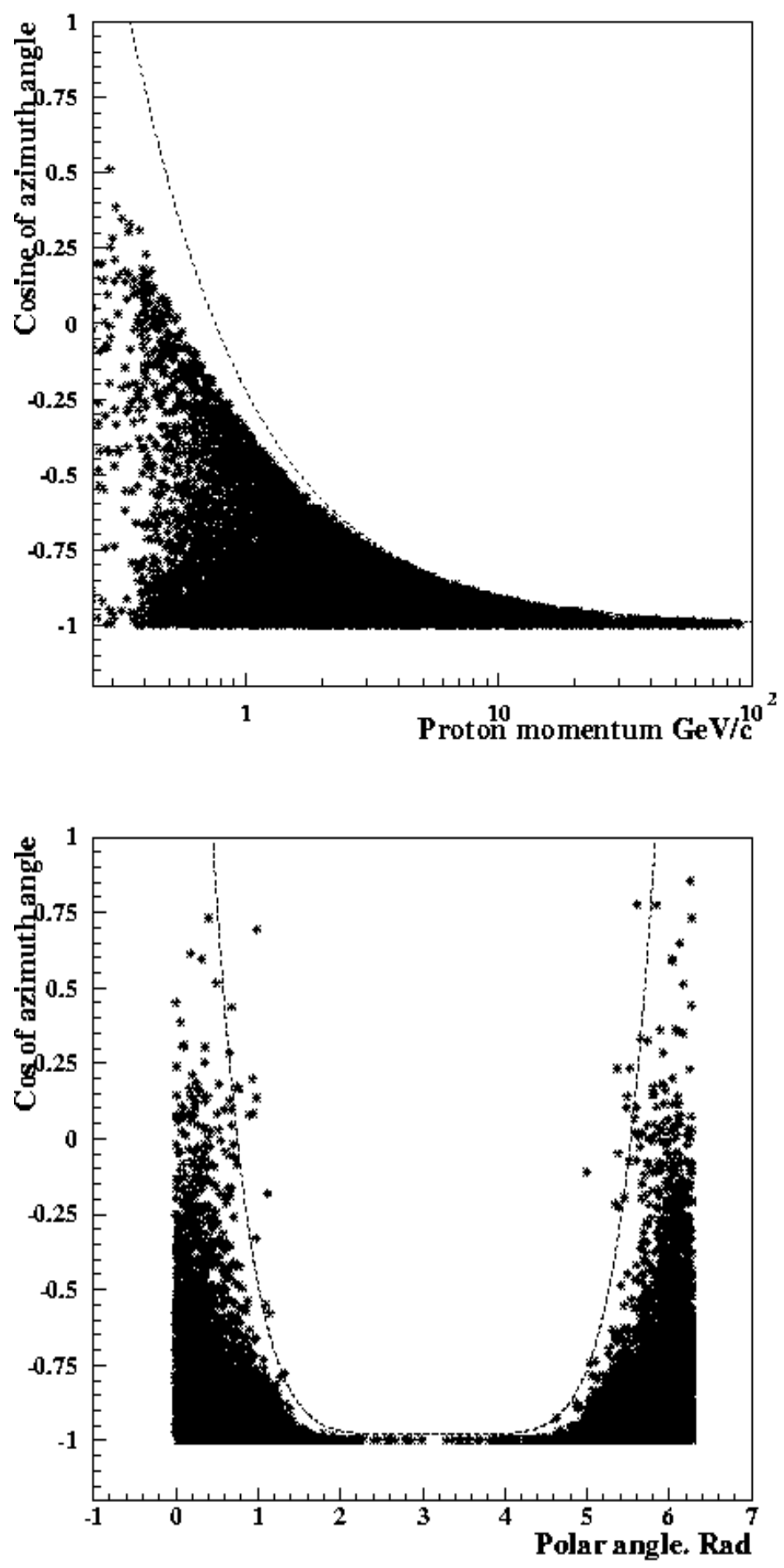

Figure 2: The phase space in $\cos \left(\Theta_{\text {emission }}\right) /$ momentum (top) and $\cos \left(\Theta_{\text {emission }}\right) / \phi_{\text {emission }}$ (bottom) planes of particles reaching the Earth's vicinity. The dashed lines show the limits used in the simulation. 


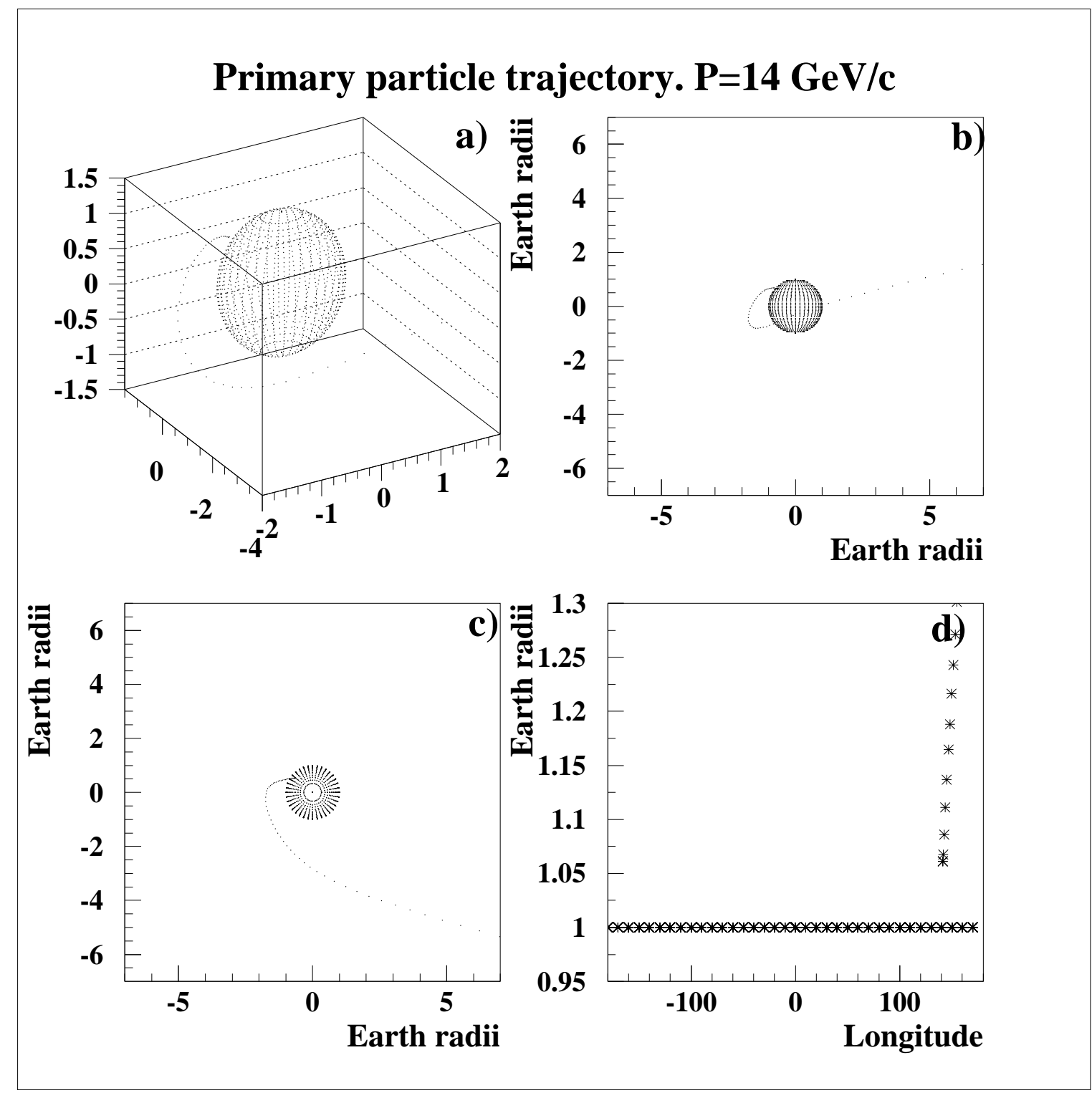

Figure 3: Primary proton trajectory in the Earth's magnetic field for a particle reaching the Earth. a) General view, b) View from 90 degree west c) View from the north pole, d) View at the Earth approach. 


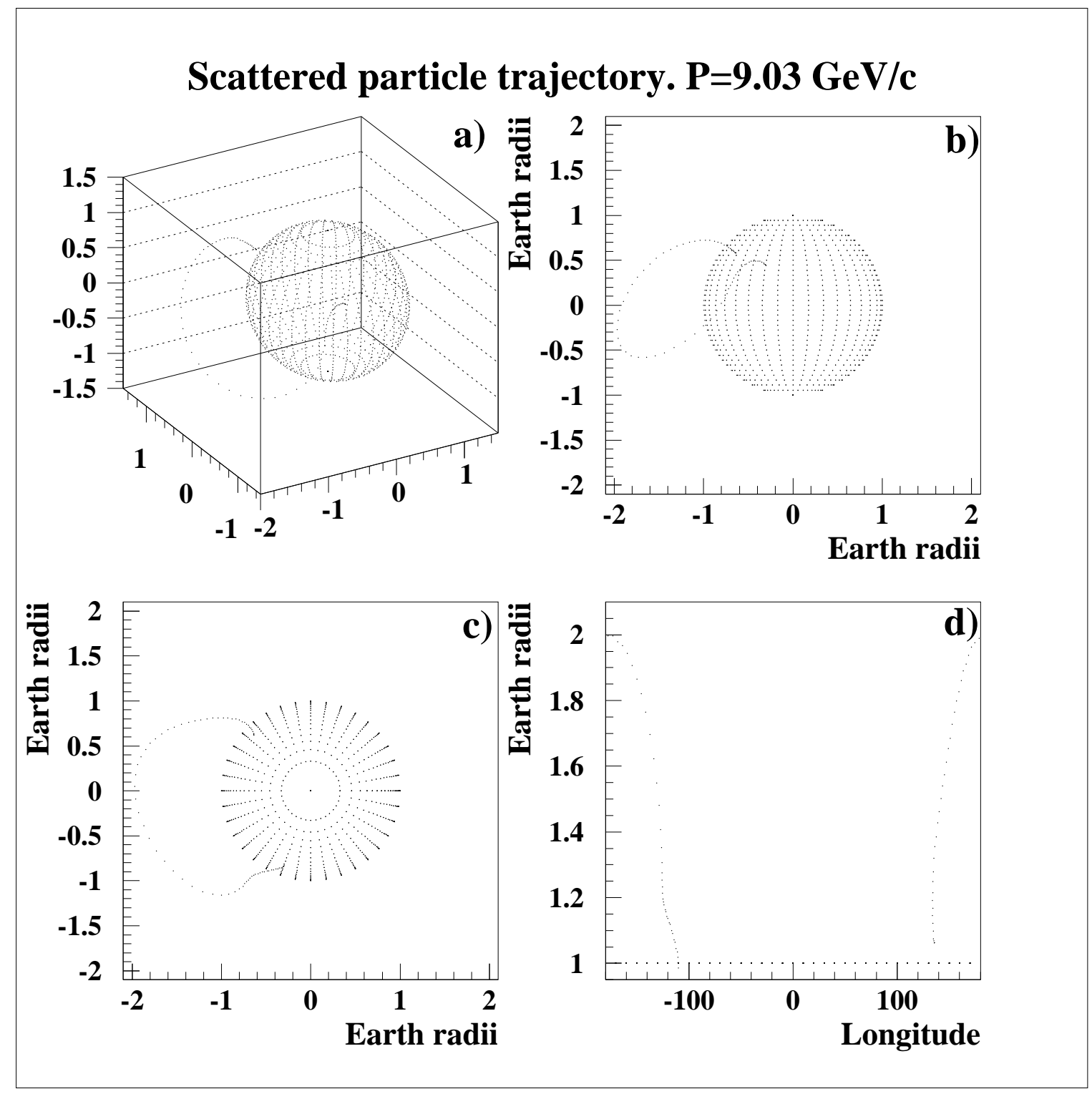

Figure 4: Trajectory of proton scattered in the atmosphere. (a) General view. (b) View from 90 degree west. (c) View from the north pole. (d) View of the scattering point and the Earth approach. 


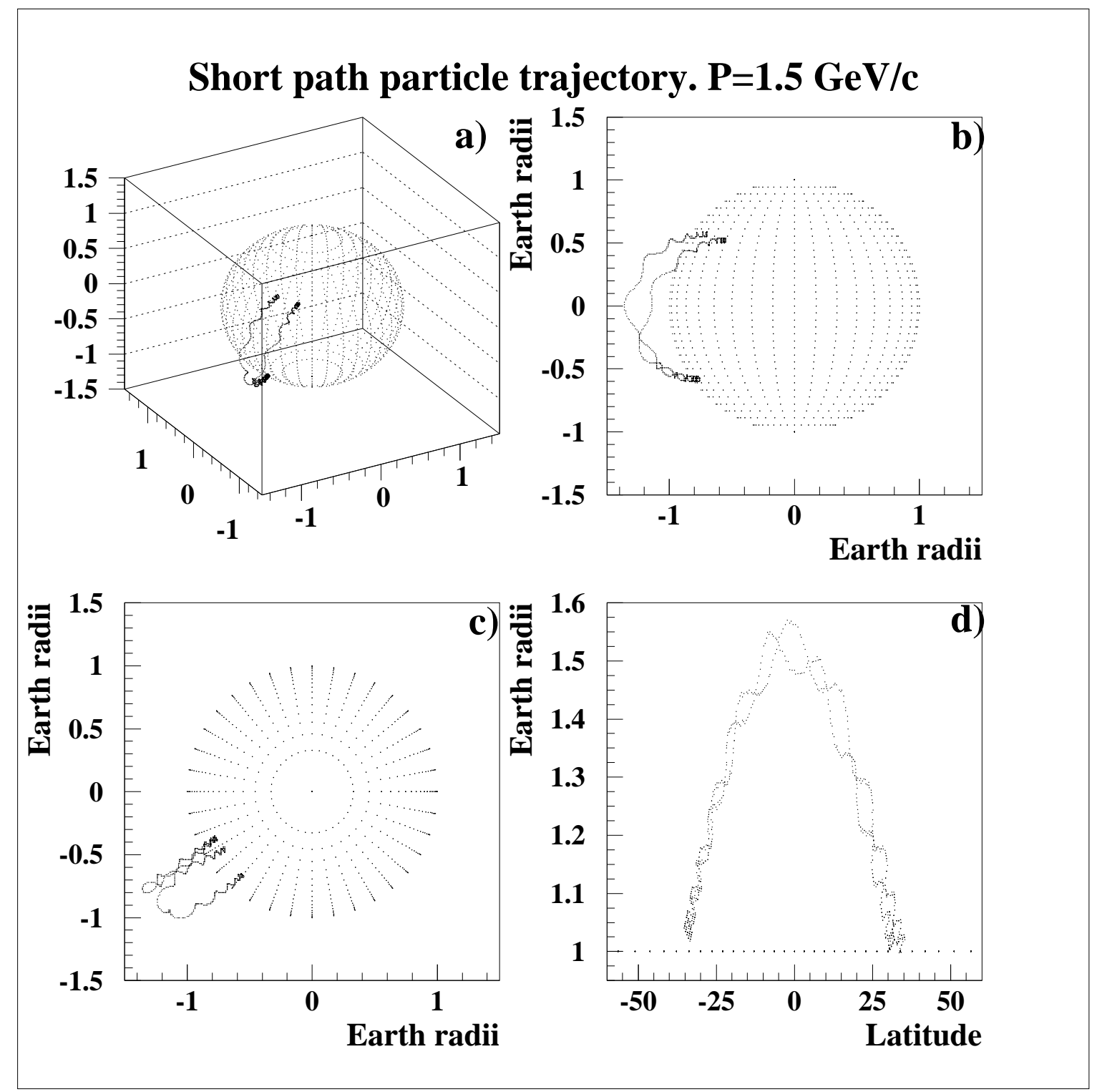

Figure 5: Trajectory of secondary positive particle with short path length. (a) General view. (b) View from 90 degree west. (c) View from the north pole. (d) Altitude at different latitude. 

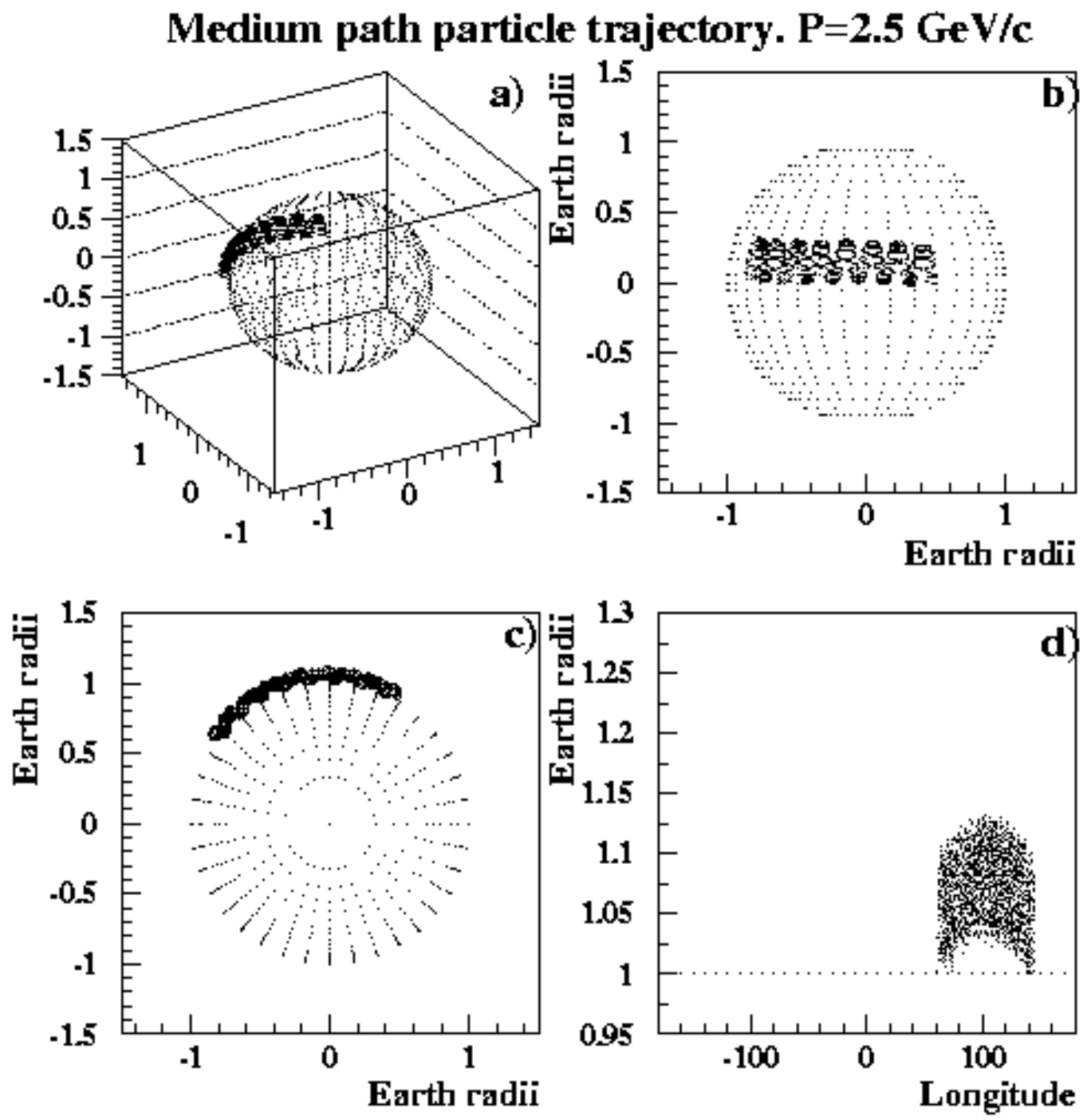

Figure 6: Trajectory of multi bouncing secondary particle with medium path length. (a) General view. (b) View from 90 degree west. (c) View from the north pole. (d) Altitude at different longitude. 

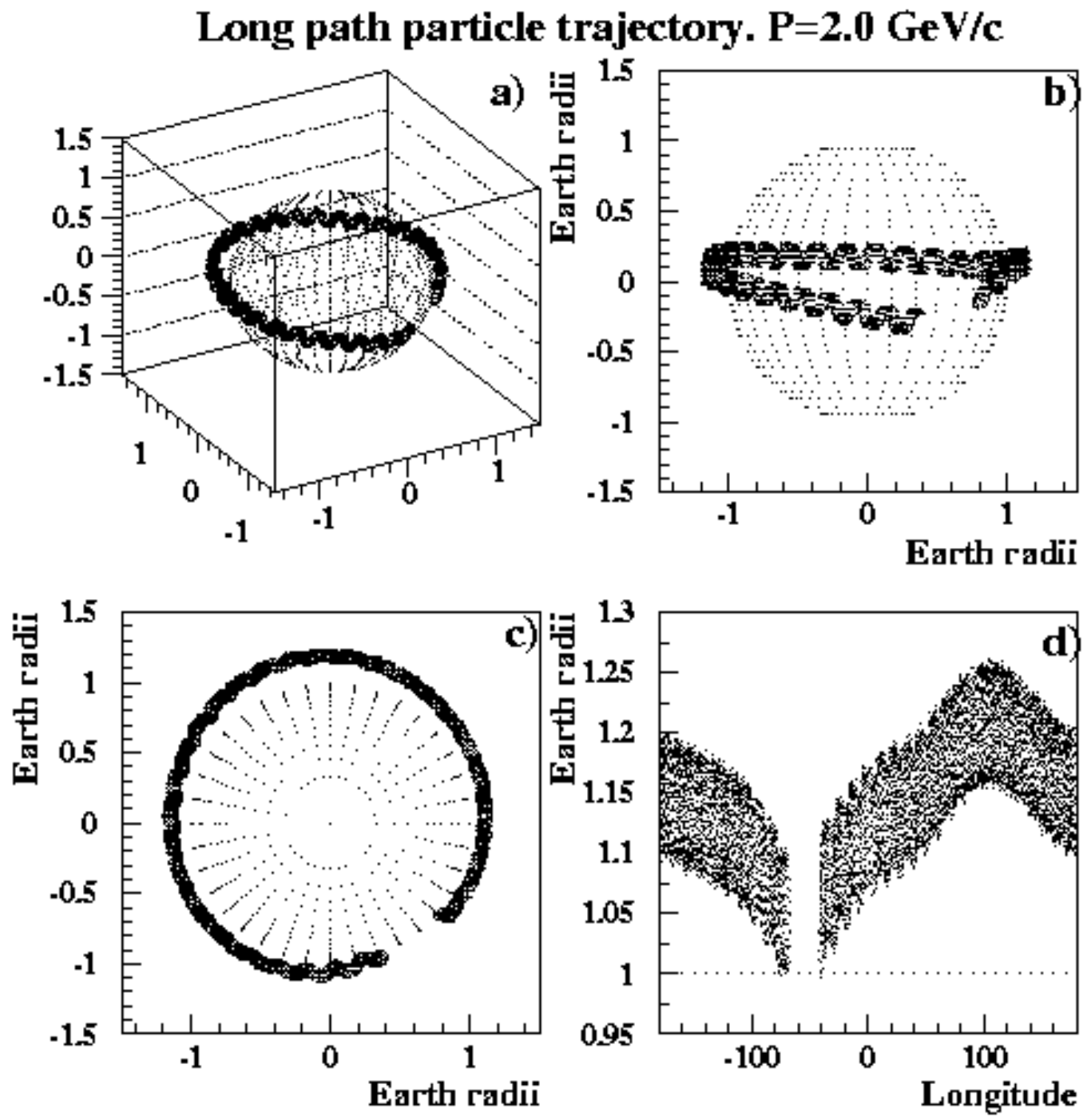

Figure 7: Trajectory of multi bouncing secondary particle with long path length. (a) General view. (b) View from 90 degree west. (c) View from the north pole. (d) Altitude at different longitude. 


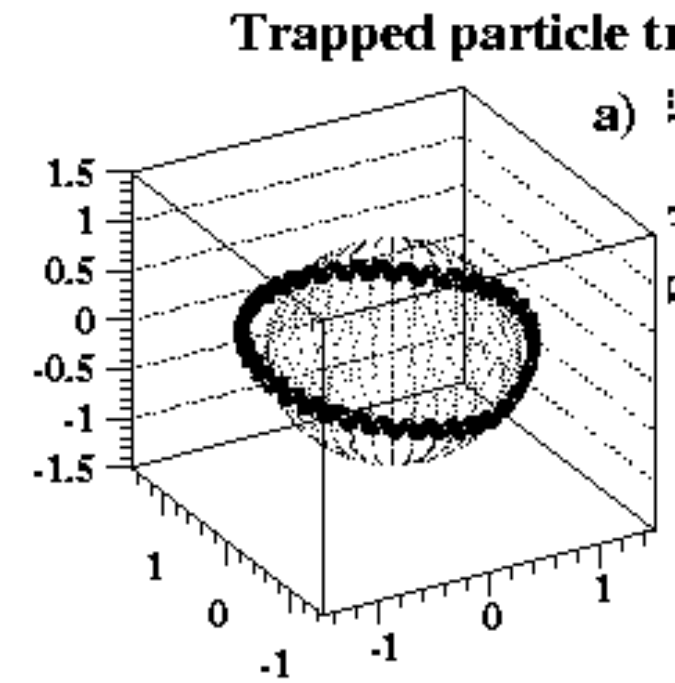

trajectory. $\mathrm{P}=1.65 \mathrm{GeV} / \mathrm{c}$
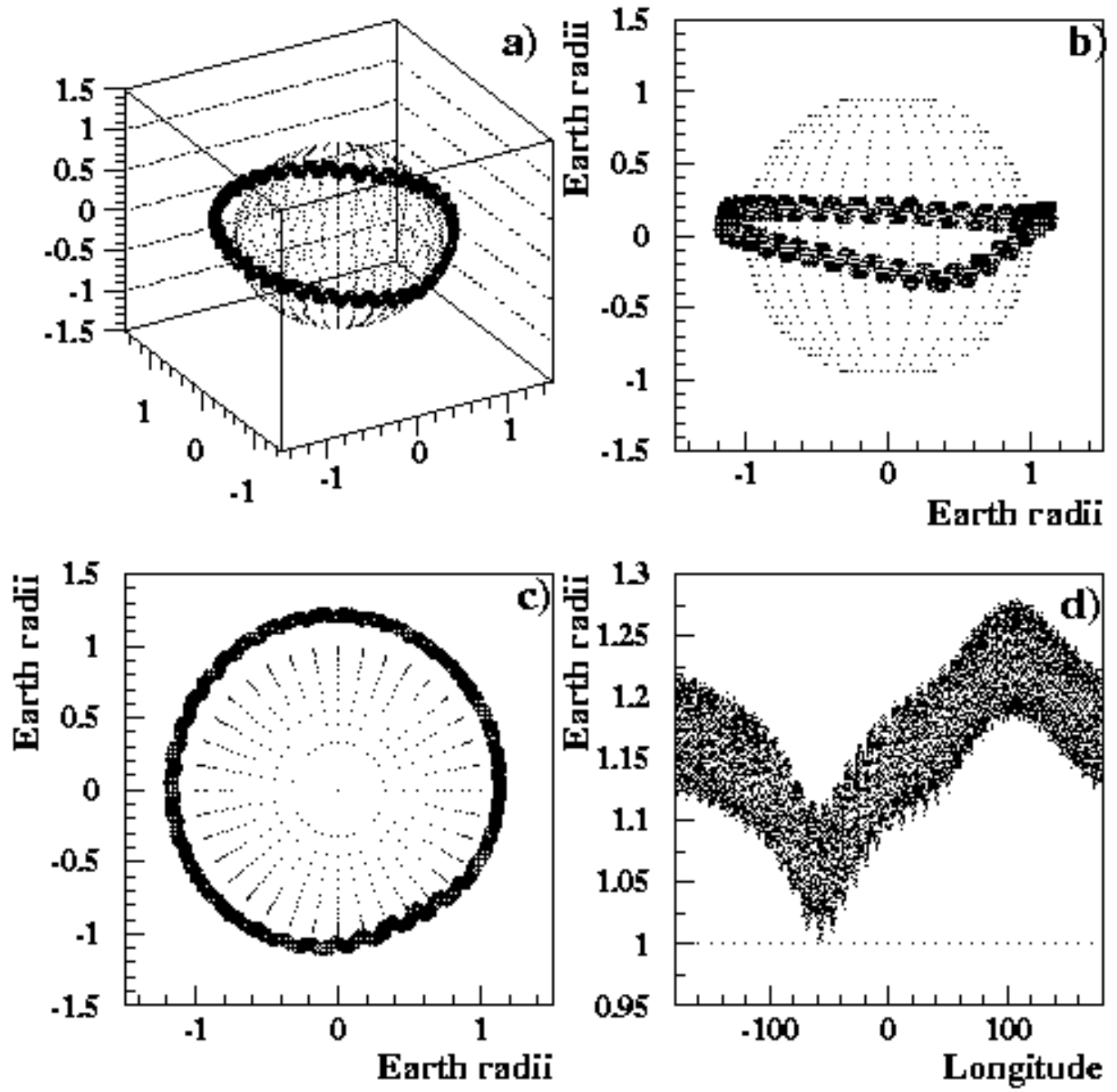

Figure 8: Closed orbit trajectory of multi bouncing secondary particle. (a) General view. (b) View from 90 degree west. (c) View from the north pole. (d) Altitude at different longitude. 
One of the consequences of the fact that in its motion the same particle can cross a detector several times is that the measured flux of such upgoing and downgoing particles is the same. A build up of the measured rate, especially in the region close to the magnetic equator, connected with the multiple crossing of the detector plane rate, does not produce secondary particles unless the spiraling particle passes through and interacts with the atmosphere. The motion of the particle being determined by the Earth's magnetic field, negative particles travel along the same trajectories as the positive ones but in the opposite direction.

The production of secondary pions and protons resulting from the interactions of cosmic (mostly He) nuclei with the atmosphere is treated using the superposition approximation [21].

The calculation presented below takes into account all effects connected with production, propagation, interaction and decay of all particles of the atmospheric shower.

\section{Particle flux}

The results of Monte Carlo simulation presented in the following correspond to a statistics of $\sim 1.5 * 10^{10}$ protons isotropically emitted from a sphere of 10 Earth's radii. This is equivalent to a $0.62 \mathrm{ps}$ exposure.

The dependence on the geographical coordinates of the rate of protons crossing a sphere surrounding the Earth at the altitude of $400 \mathrm{~km}$ is shown in Figure 9 for downward (top picture) and upward (bottom picture) protons. The rate includes all i.e. primary and secondary, protons and is averaged over all directions in the corresponding hemispheres.

A typical picture with high rate near the magnetic poles and the depleted by the magnetic cutoff regions near the magnetic equator is clearly seen in the downward proton rate behaviour. The geographical pattern of upgoing protons is similar in shape but at much lower particle rate.

The flux of protons going toward the Earth (Figs 10 and b) consists of original protons from space i.e. those with $p / p_{\text {init }}>0.9999996$, where $p_{\text {init }}$ is the initial cosmic proton momentum and $p$ is the momentum of the proton crossing the detection sphere (see Fig (3), protons scattered in the atmosphere $\left(0.995<p / p_{\text {init }}<0.9999996\right.$. Fig 4 $)$ and secondary protons $\left(p / p_{\text {init }}<0.995\right.$. Figs 5678). The flux of upgoing protons (Figs 10p and d) contains reflected protons most of which have lost some energy in the atmosphere as well as scattered protons on their way from the Earth and the secondary protons. 


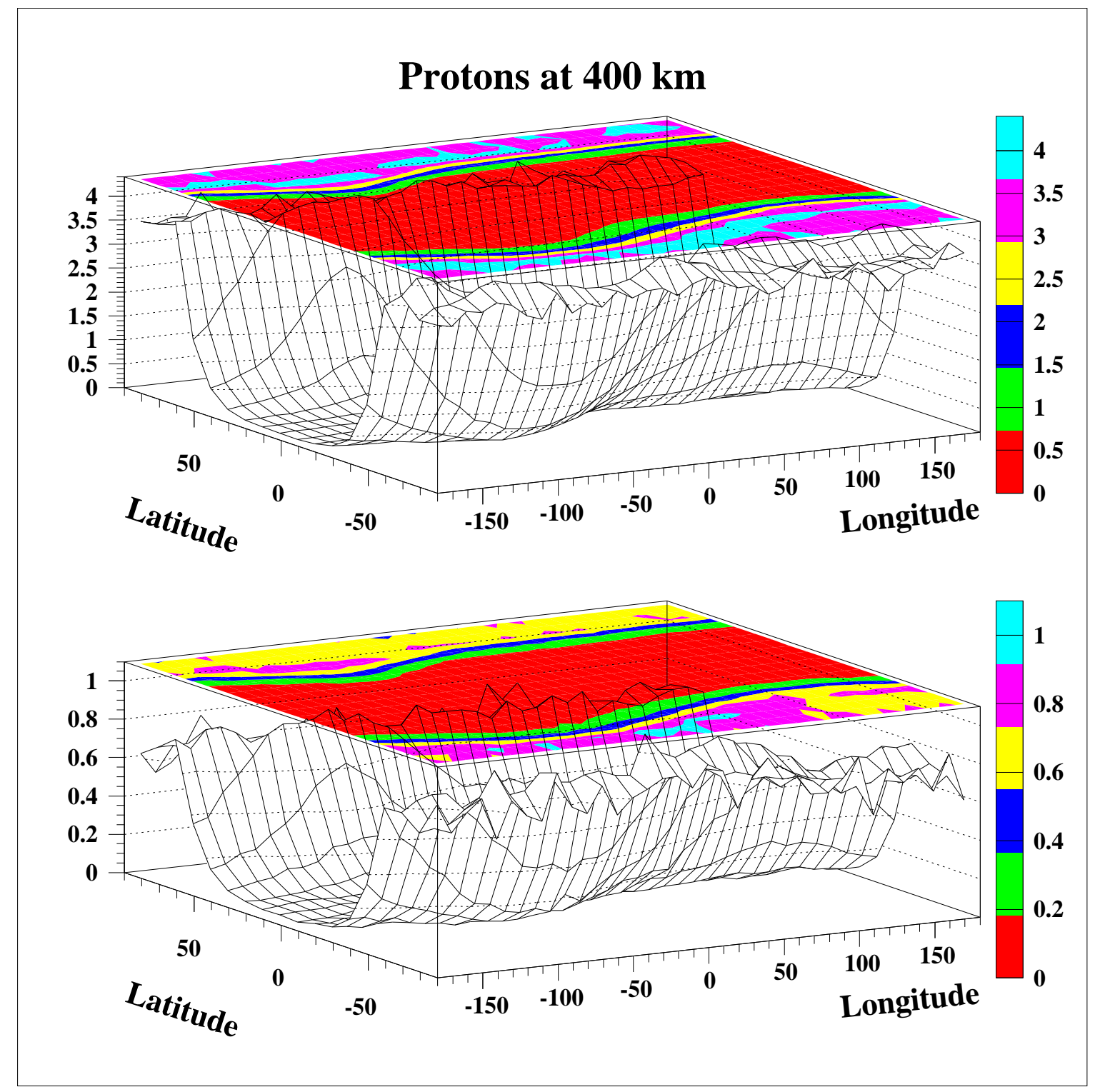

Figure 9: Proton rate (in $\mathrm{KHz} / \mathrm{m}^{2} \mathrm{sr}$ ) at $400 \mathrm{~km}$ altitude averaged over all directions. Top: earthward protons, bottom: spaceward protons. 


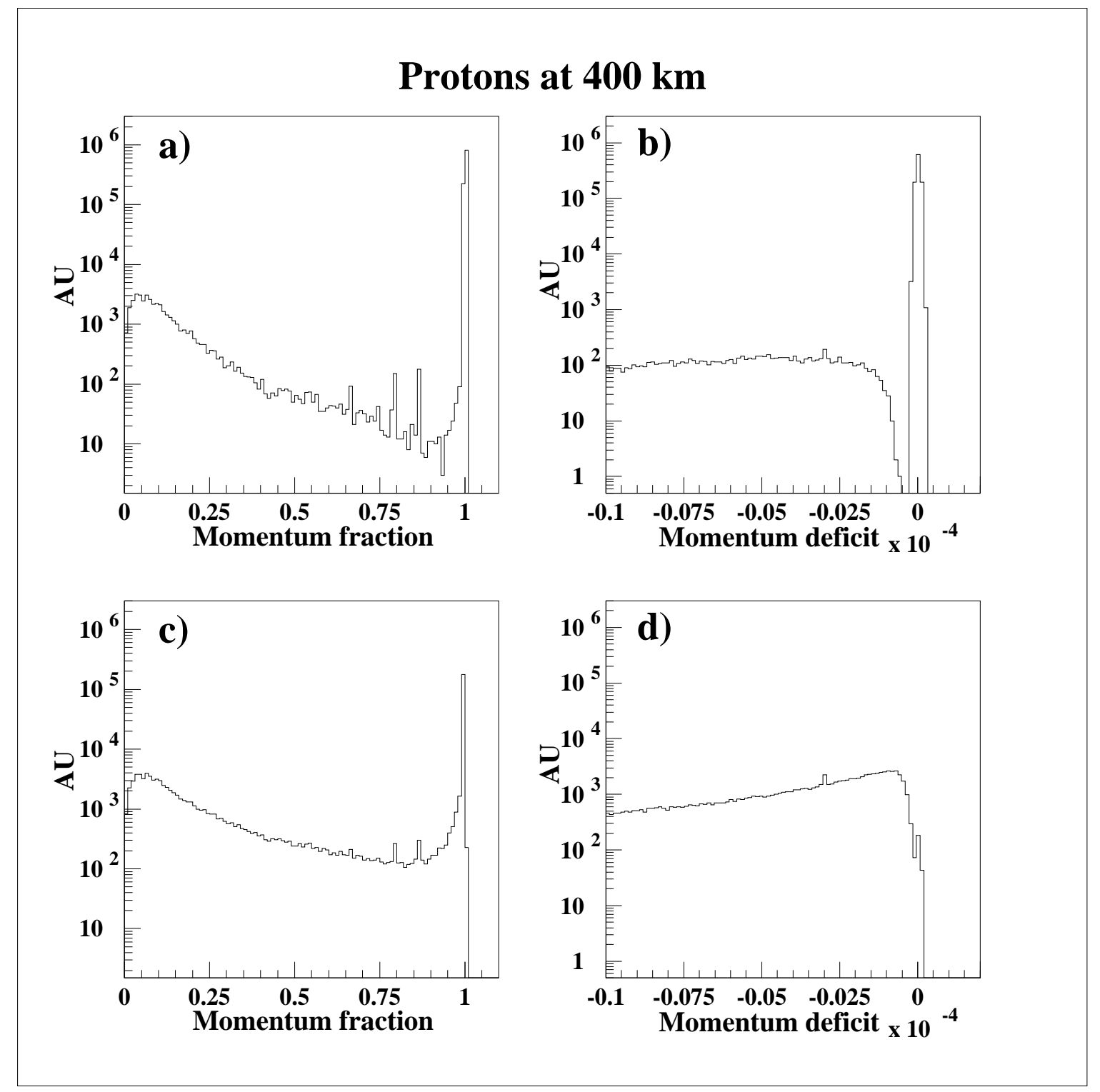

Figure 10: Partial (with respect to initial proton) momentum of protons detected at 400 km. (a) All downgoing protons. (b) Close up on the close to unity region of (a). (c) All upgoing protons. (d) Close up on the close to unity region of (c). 
The important feature of the primary flux relevant to neutrino production is that up to momenta as high as $\sim 40 \mathrm{GeV}$ it becomes anisotropic for directions close to parallel to the Earth's surface. (see Fig[11). This decrease is not in contradiction with the AMS measurement because the orientation of the AMS detector during the flight did not allow to measure the horizontal flux.

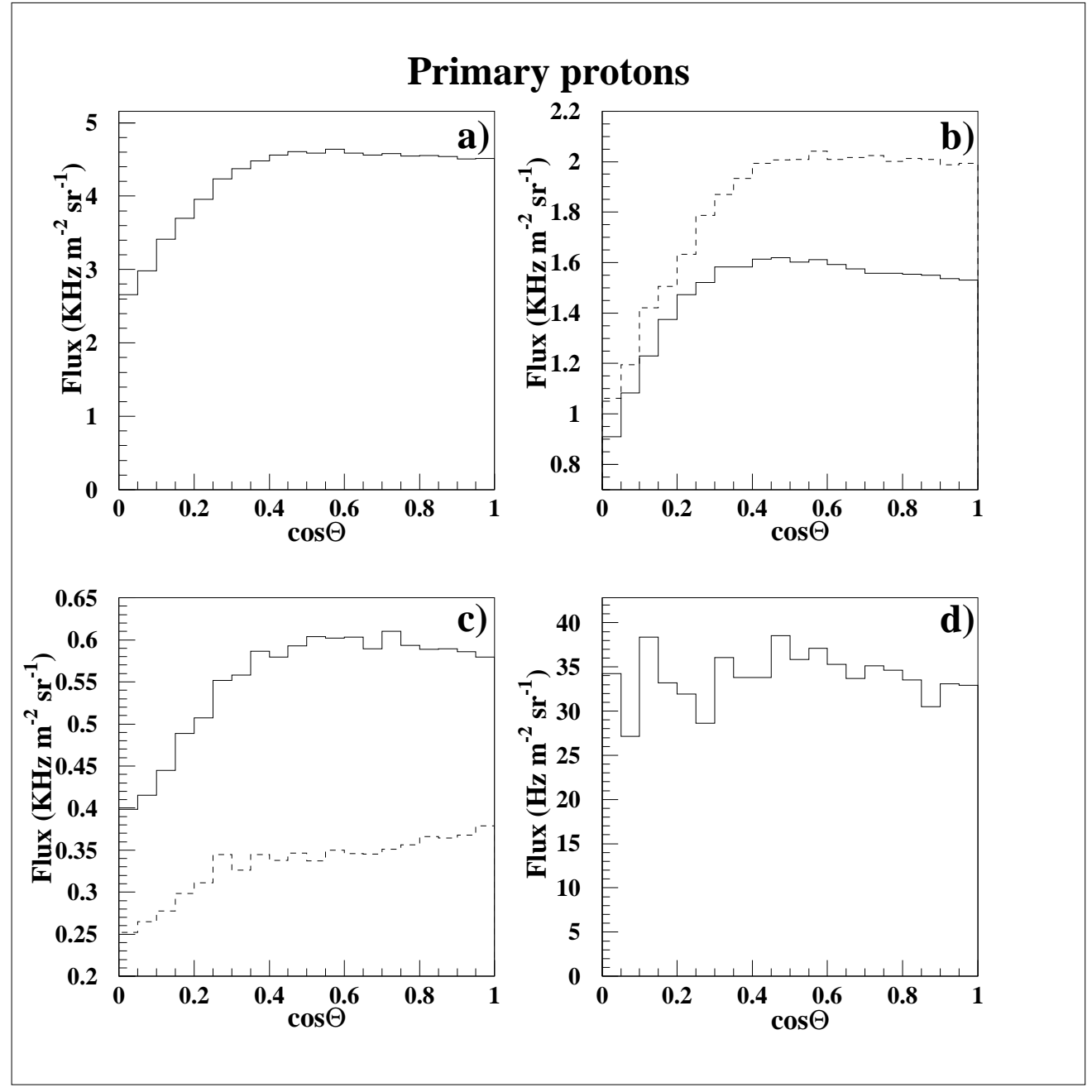

Figure 11: Dependence of primary proton flux at $400 \mathrm{~km}$ on the proton incidence angle with respect to nadir. (a) All momenta. (b) Solid line: $p<1.5 \mathrm{GeV} / \mathrm{c}$, dashed line: $1.5<$ $p<5.0 \mathrm{GeV} /$ c. (c) Solid line:5.0 $<p<10.0 \mathrm{GeV} /$ c, dashed line: $10.0<p<40.0 \mathrm{GeV} / \mathrm{c}$. (d) $\mathrm{p}>40 . \mathrm{GeV} / \mathrm{c}$. 
Figure 12 illustrates the behaviour of the flux of primary protons. Figure 12a shows the dependence of the cutoff on the magnetic latitude. The cutoff momentum is about $10 \mathrm{GeV} / \mathrm{c}$ at the equator and goes down to zero at the poles. One can see, however, that there are some under cutoff particles even in the flux of primary protons. These under cutoff particles are present at all magnetic latitudes (Figs 12 a and d). The other feature of these protons is that they are moving predominantly, and in the magnetic equator region exlusivly (Figs 12b and c), toward the Earth.

The scattered protons (Fig[13) are mostly detected in the regions of the flux maxima close to the magnetic poles (Fig 13a). These are mostly albedo protons (Figs 13b and $\mathrm{d}$ ) although there is a considerable amount of those (Figs 13b and c) reentering the atmosphere (see fig 4 ).

Secondary protons (Fig 14) produced at the magnetic poles add up to the albedo flux (Fig 14b) whereas those produced in the regions closer to the equator follow the trajectories illustrated in Figs 567, 8 producing equal fluxes of upgoing and downgoing undercutoff particles (Fig 14b). The impact points of particles with multiple crossing of the detection sphere are clearly seen in Fig 14 . The rate of under cutoff protons in the magnetic equator region is dominated by the long traveling protons (see Figs 67]).

Positrons and electrons resulting from the decays of secondary pions display the behaviour similar to that of secondary protons allowing for much faster energy losses in the atmosphere due to the difference in radiation and nuclear interaction lengths.

\section{$5 \quad$ Particles detected by zenith facing AMS}

To compare the results of calculations with the AMS measurements [24, 25] the overall fluxes produced in the simulation were restricted to the AMS acceptance during the zenith facing flight period. [23, 39]. In accordance with the AMS construction no particle with kinetic energy below $\sim 120 \mathrm{MeV}$ can trigger AMS. The AMS orbit spans within the geographic latitude of $\pm 51.7^{\circ}$. The corresponding cuts are applied on the MC simulated data. The result of Monte Carlo is an absolute one. No renormalization is applied to make a comparison with the data. As in AMS publications the spectra are presented as a function of kinetic energy of the particles. Figure [15 shows the spectra of protons obtained in the simulation for different positions of AMS with respect to the magnetic equator. The result of simulation correctly reproduces the characteristic features present in the AMS data (black dots in the figure) such as:

- the value of the cutoff at different magnetic latitudes;

- the presence of protons with under cutoff momenta at all magnetic latitudes;

- the equality of fluxes of under cutoff protons going to and from the Earth; 


\section{Primary protons}
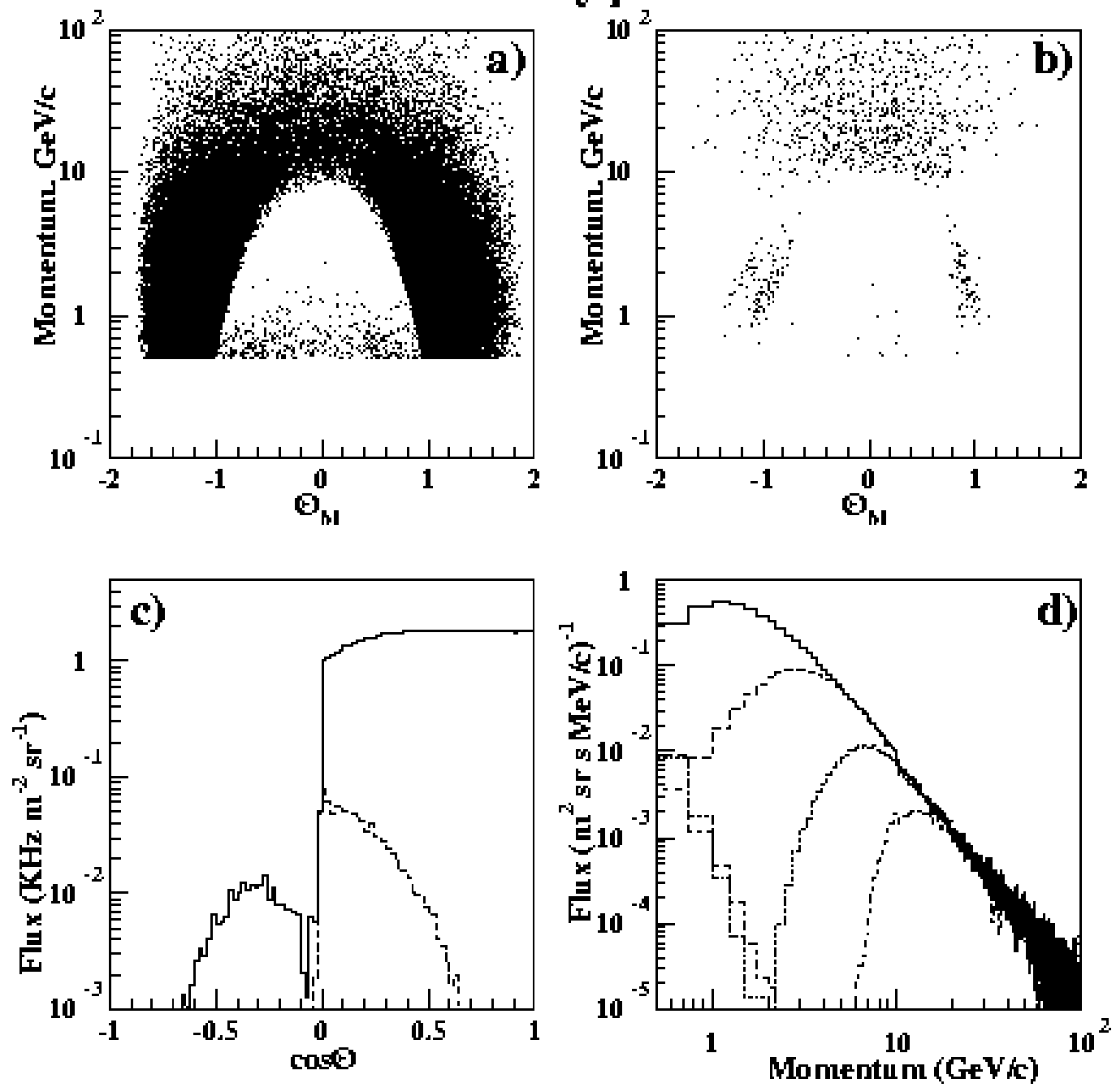

Figure 12: Primary protons detected at $400 \mathrm{~km}$. Momentum at different magnetic latitude for (a) downgoing and (b) upgoing protons. (c) Average flux at different angles with respect to nadir. Solid line - all primary protons, dashed line - only under cutoff protons in the equator $\left(\left|\Theta_{M}\right|<0.9\right)$ region. (d) Momentum spectra at different magnetic latitudes $\left(\Theta_{M}\right)$. Solid line $-0.9<\Theta_{M}<1.1$, dashed line $0.7<\Theta_{M}<0.9$, dotted line $-0.4<\Theta_{M}<0.7$, dashed-dotted line $-\left|\Theta_{M}\right|<0.4$ 


\section{Scattered protons}
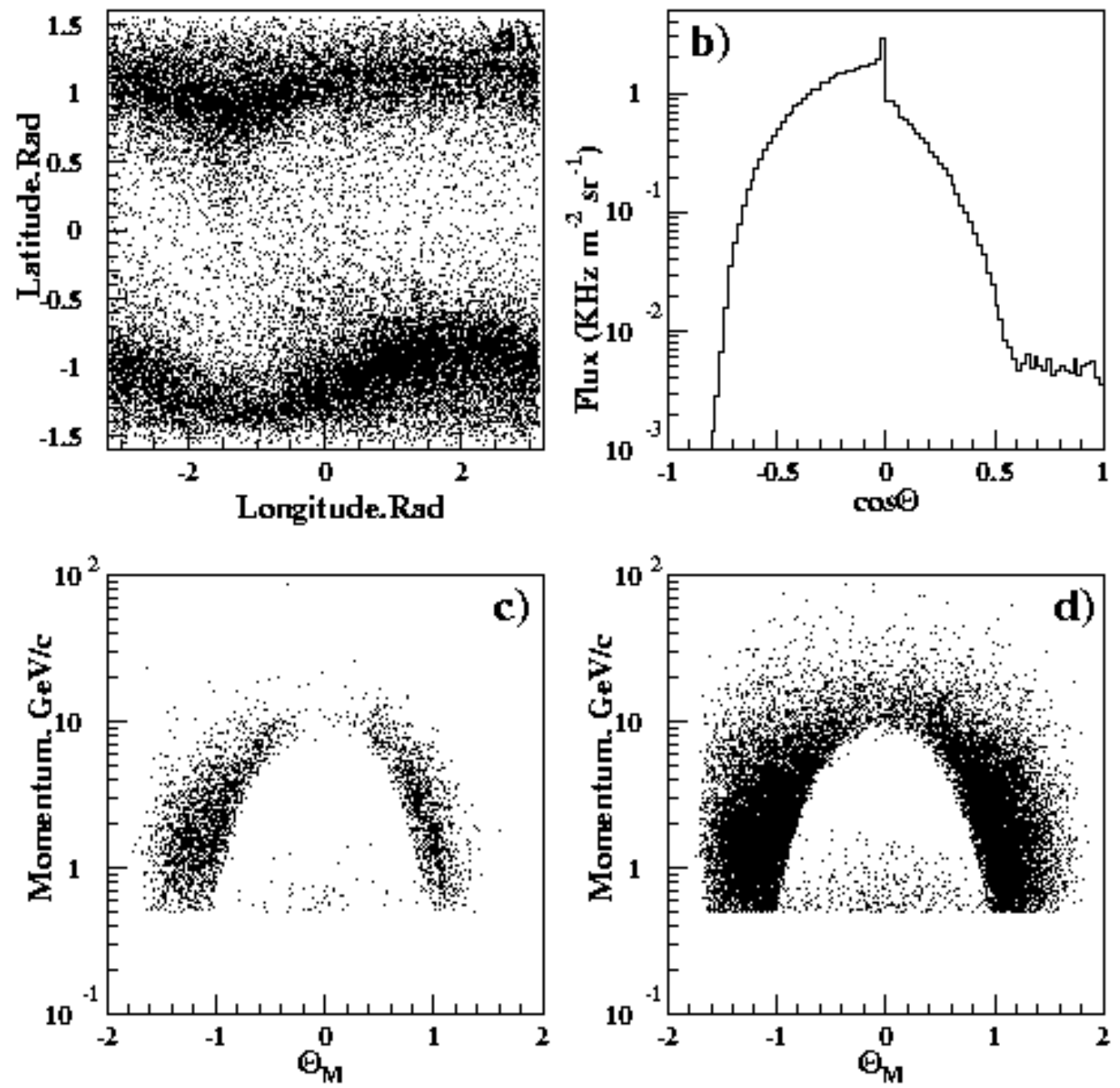

Figure 13: Scattered protons detected at $400 \mathrm{~km}$. (a) Geographic coordinates of the crossing points. (b) Average flux at different angles with respect to nadir. Momenta dependence on magnetic latitude for (c)- downgoing and (d) - upgoing protons. 


\section{Secondary protons}
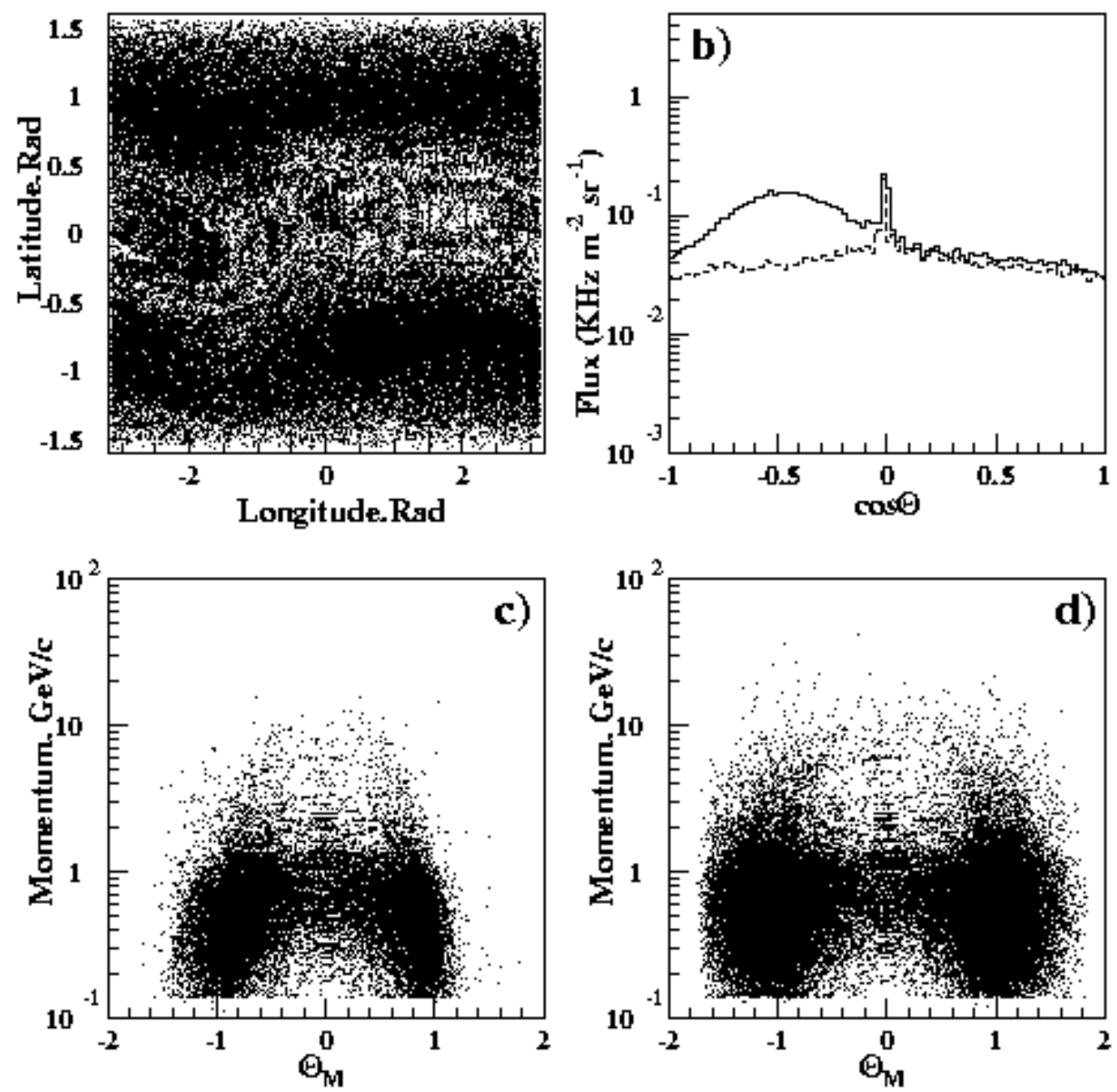

Figure 14: Secondary protons detected at $400 \mathrm{~km}$. (a) Geographic coordinates of the crossing points (b) Avergae flux at different angles with respect to nadir for pole $\left(\left|\Theta_{M}\right|>0.7\right.$ $\mathrm{rad}$, solid line) and equator $\left(\left|\Theta_{M}\right|<0.7 \mathrm{rad}\right.$, dashed line) regions, momentum dependence on magnetic latitude for (c)- downgoing and (d) - upgoing protons. 


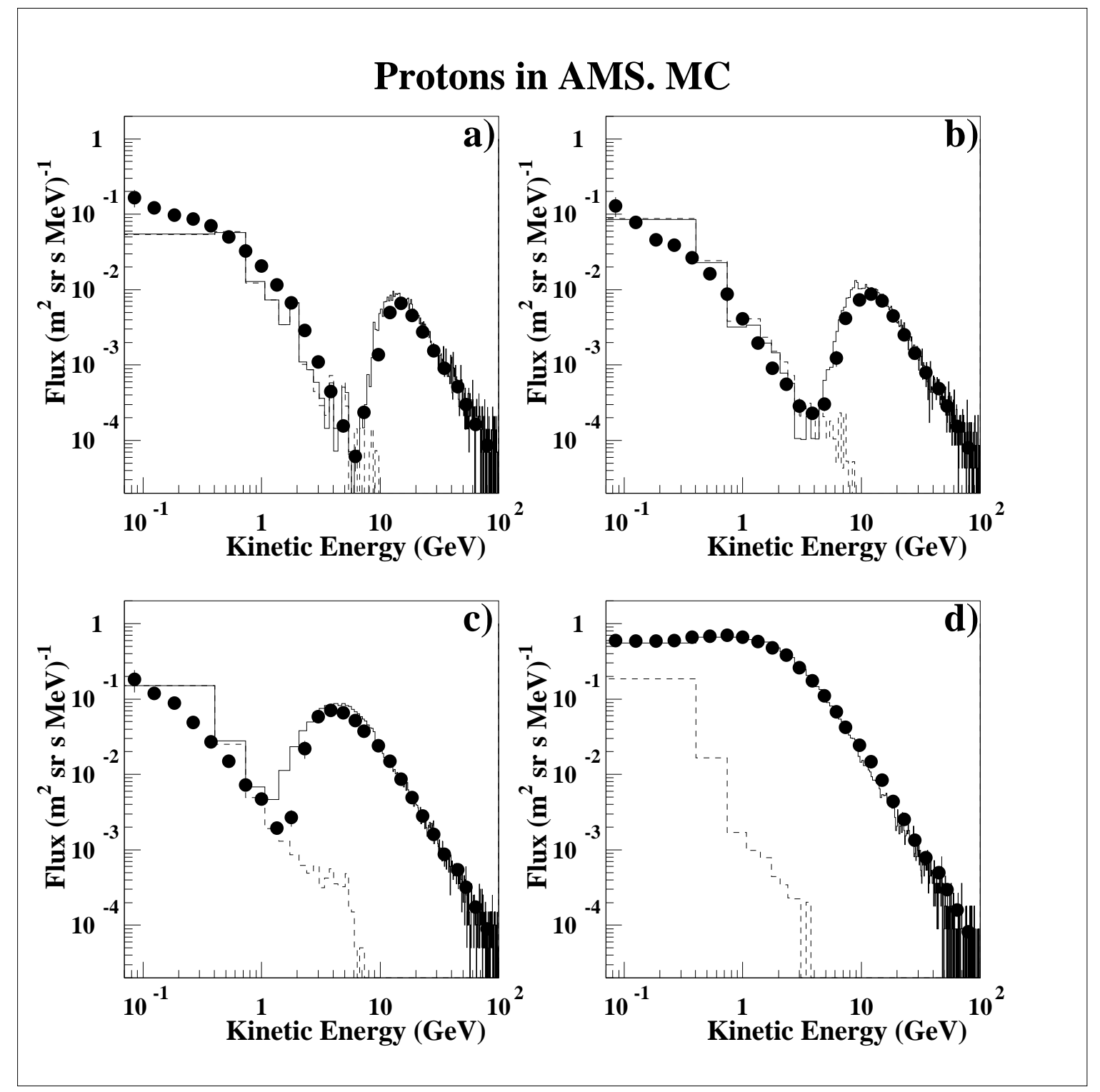

Figure 15: Proton flux in AMS at different magnetic latitudes $\left(\left|\Theta_{M}\right|\right)$. Solid line - flux from the top, dashed line - flux from the bottom. a) $\left|\Theta_{M}\right|<0.2 \mathrm{rad}$, b) $0.2<\left|\Theta_{M}\right|<0.5$ $\left.\mathrm{rad}, \mathrm{c}) 0.5<\left|\Theta_{M}\right|<0.8 \mathrm{rad}, \mathrm{c}\right) 0.8<\left|\Theta_{M}\right|<1.1 \mathrm{rad}$. The dots is the AMS measurement. 


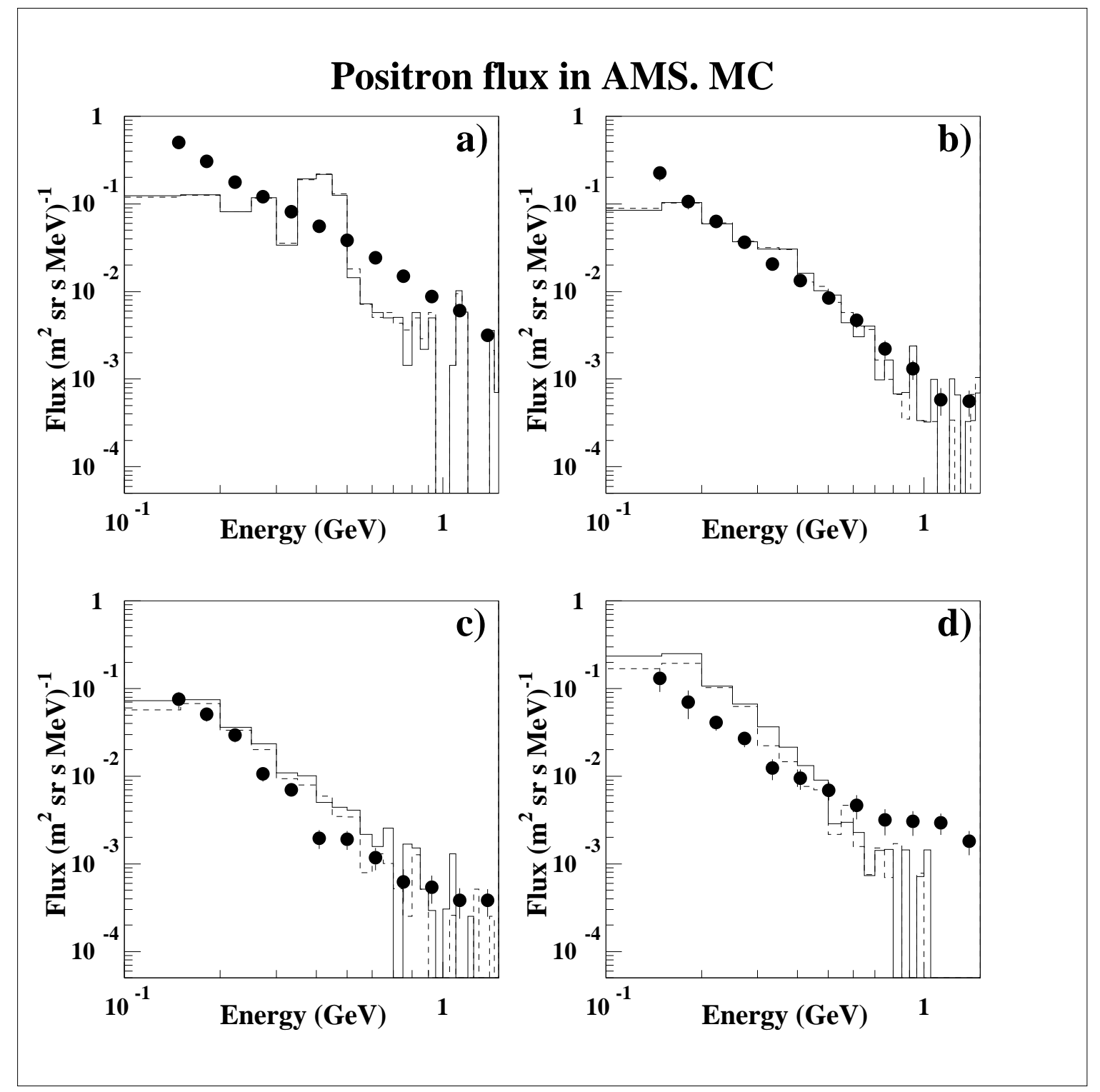

Figure 16: Secondary positron flux in AMS at different magnetic latitudes $\left(\left|\Theta_{M}\right|\right.$. Solid line - flux from the top, dashed line - flux from the bottom. a) $\left|\Theta_{M}\right|<0.3 \mathrm{rad}$, b) $0.3<\left|\Theta_{M}\right|<0.6 \mathrm{rad}$, c) $0.6<\left|\Theta_{M}\right|<0.8 \mathrm{rad}$, c) $0.8<\left|\Theta_{M}\right|<1.1 \mathrm{rad}$. The dots is the AMS measurement. The enhancement at higher energies of the measured flux at high magnetic latitude is due to primary cosmic positrons detected at the lower magnetic cutoff region. 


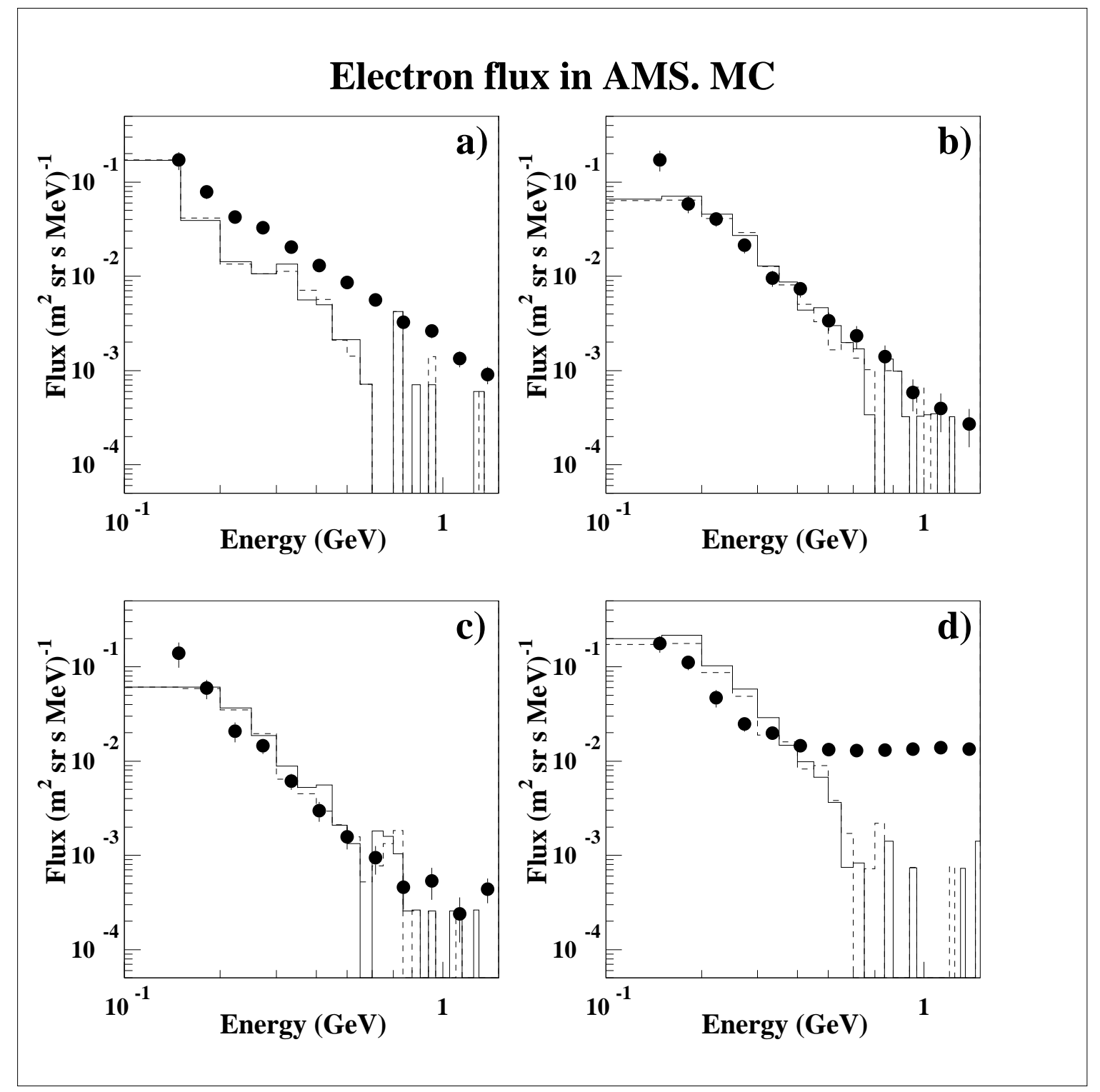

Figure 17: Secondary electron flux in AMS at different magnetic latitudes $\left(\left|\Theta_{M}\right|\right.$. Solid line - flux from the top, dashed line - flux from the bottom. a) $\left|\Theta_{M}\right|<0.3 \mathrm{rad}$, b) $0.3<\left|\Theta_{M}\right|<0.6 \mathrm{rad}$, c) $0.6<\left|\Theta_{M}\right|<0.8 \mathrm{rad}$, d) $0.8<\left|\Theta_{M}\right|<1.1 \mathrm{rad}$. The dots is the AMS measurement. The enhancement at higher energies of the measured flux at high magnetic latitude is due to primary cosmic electrons detected at the lower magnetic cutoff region. 


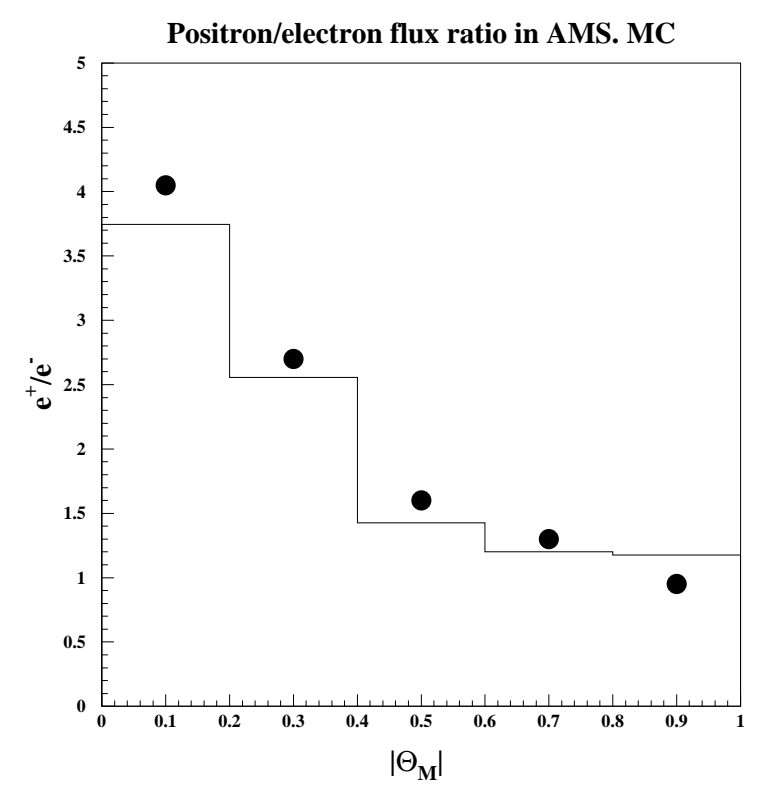

Figure 18: Calculated dependence on magnetic latitude of positron/electron flux ratio. The dots is the AMS measurement.

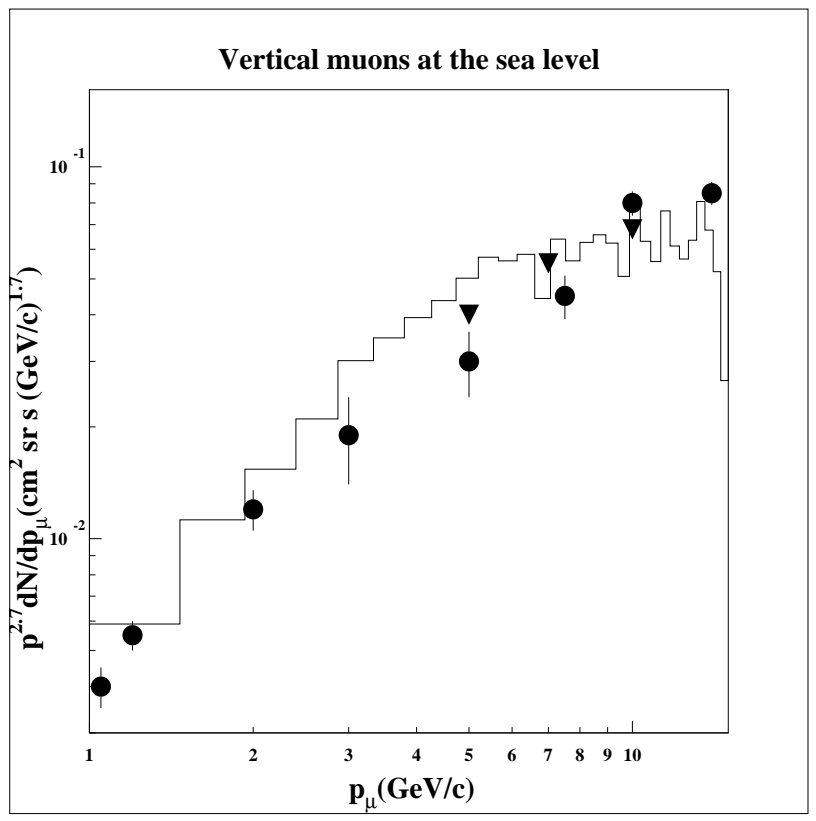

Figure 19: Vertical muon flux at the sea level. Histrogram: this study. Circles: data from Ref. 44], triangles: data from Ref. 45. 
- the energy spectra of under cutoff protons and their absolute flux.

Figures [16] and [17 show the spectra of secondary positrons and electrons correspondingly. Although the positrons and electrons can not contribute to atmospheric neutrino production they are (together with cosmic muons) the "spectators" of neutrino production in the decays of particles in the atmospheric showers and consequently the correct prediction of the fluxes of these particles is a proof of validity of the whole simulation procedure including the neutrino flux predictions. The simulated positron and electron spectra are similar to those obtained in the AMS experiment [25] for the secondary leptons in terms of equality of fluxes of upgoing and downgoing particles and energy dependence of the flux at different magnetic latitudes.

The difference in the measured and simulated secondary leptonic (and to some extent secondary proton) fluxes at low magnetic latitudes (Figs [16, 17a and 15a) is due to insufficient statistics of the present Monte Carlo study. As mentioned above, the measured rate in this region is largerly connected with the relatively rare long traveling particles multiply crossing the AMS orbit plane. Actually, the peak in the spectrum of figure 16a is due to just 3 spiraling positrons each crossing the AMS detector plane about 500 times in both upward and downward direction. Nevertheless the measured relative rate of secondary leptons [25, 40] and their dependence on the magnetic latitude is well reproduced by the simulation (Fig[18). It is worth to emphasize that the impact of long traveling secondaries on the production of atmospheric neutrinos is negligible.

Apart from the data connected with those measured by AMS the present simulation includes a prediction of the fluxes of muons at the sea level. The histogram in Fig [19] shows a result obtained in this study for the vertical muon flux. The result is in good agreement with the measured fluxes reported in [44, 45].

\section{Atmospheric neutrino fluxes}

As for the fluxes of charged particles discussed in the previous section all results that follow are obtained directly without any renormalization. The dependence of the neutrino flux averaged over the entire Earth's surface on the neutrino direction with respect to nadir is presented in Fig 20]. The overall equality of neutrino fluxes to and from the Earth is clearly seen. The dashed line in the figure is a mirror image of the flux from the top. A small increase of the flux of almost horizontal neutrinos coming from inside the Earth is due to neutrinos produced by the cosmic particle's showers in the Earth. The relative flux of different kinds of atmospheric neutrinos $\left(\nu_{\mu}, \tilde{\nu}_{\mu}, \nu_{e}, \tilde{\nu}_{e}\right)$ is constant for all incidence angles (Fig 20b.). The averaged over all directions energy spectra of neutrino of different kind are presented in Fig 20k. The dependence of the flux on the azimuth (with respect to east direction) angle (Fig $20 \mathrm{~d})$ reflects some east-west asymmetry of average neutrino fluxes. 


\section{Overall neutrino flux}
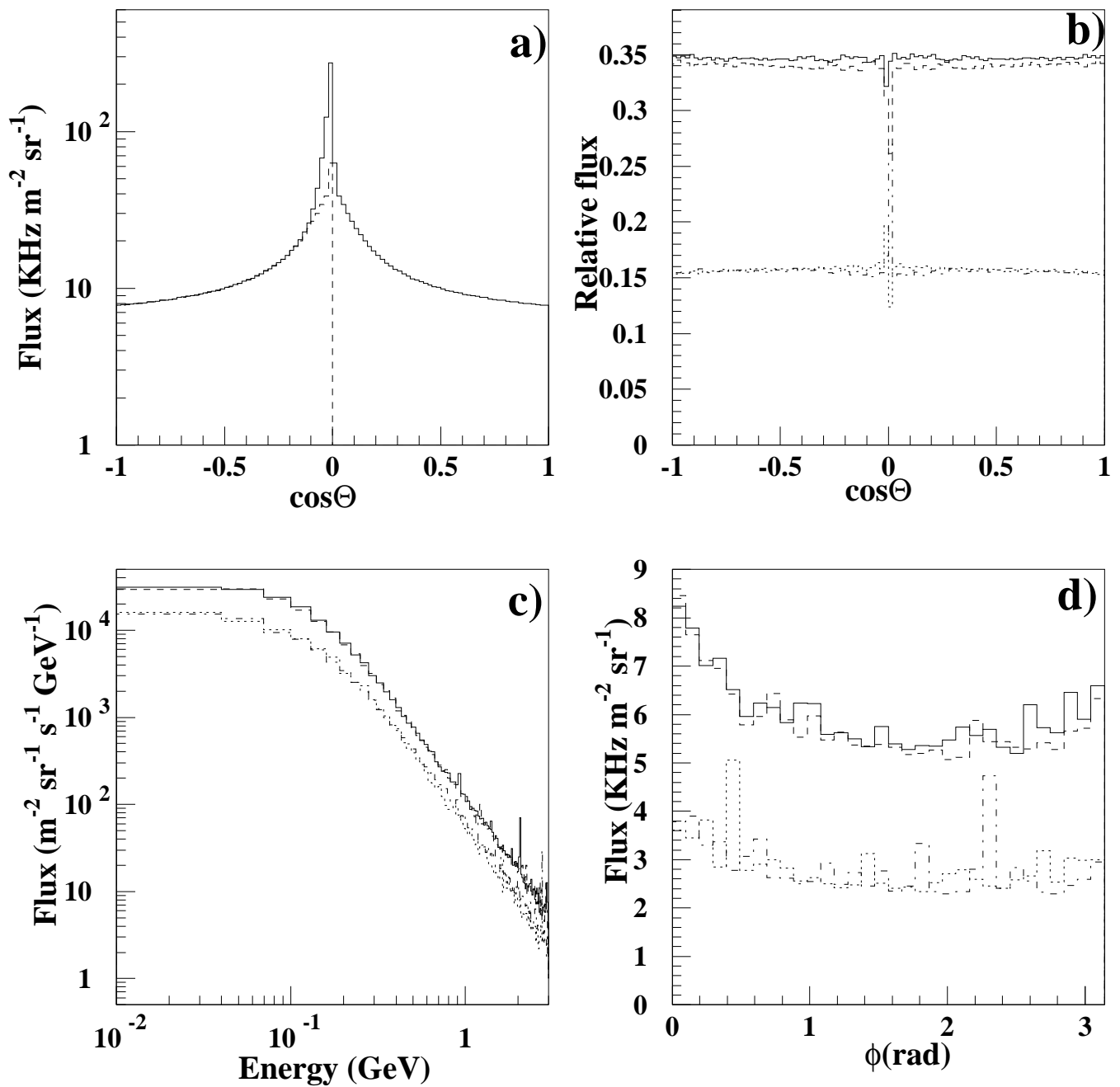

Figure 20: Neutrino flux averaged over the Earth's surface. The angle of incidence $(\Theta)$ is measured from nadir $(\cos \Theta=1$ for particles going vertically down). The dashed line in a) is a mirror image of the flux from the top. b) Relative flux of different kinds of neutrino. c) Energy spectra. d) Azimuth angle measured from direction toward east. Solid line $\tilde{\nu}_{\mu}$, dashed line $-\nu_{\mu}$, dotted line $-\tilde{\nu}_{e}$, dashed-dotted line $-\nu_{e}$. 


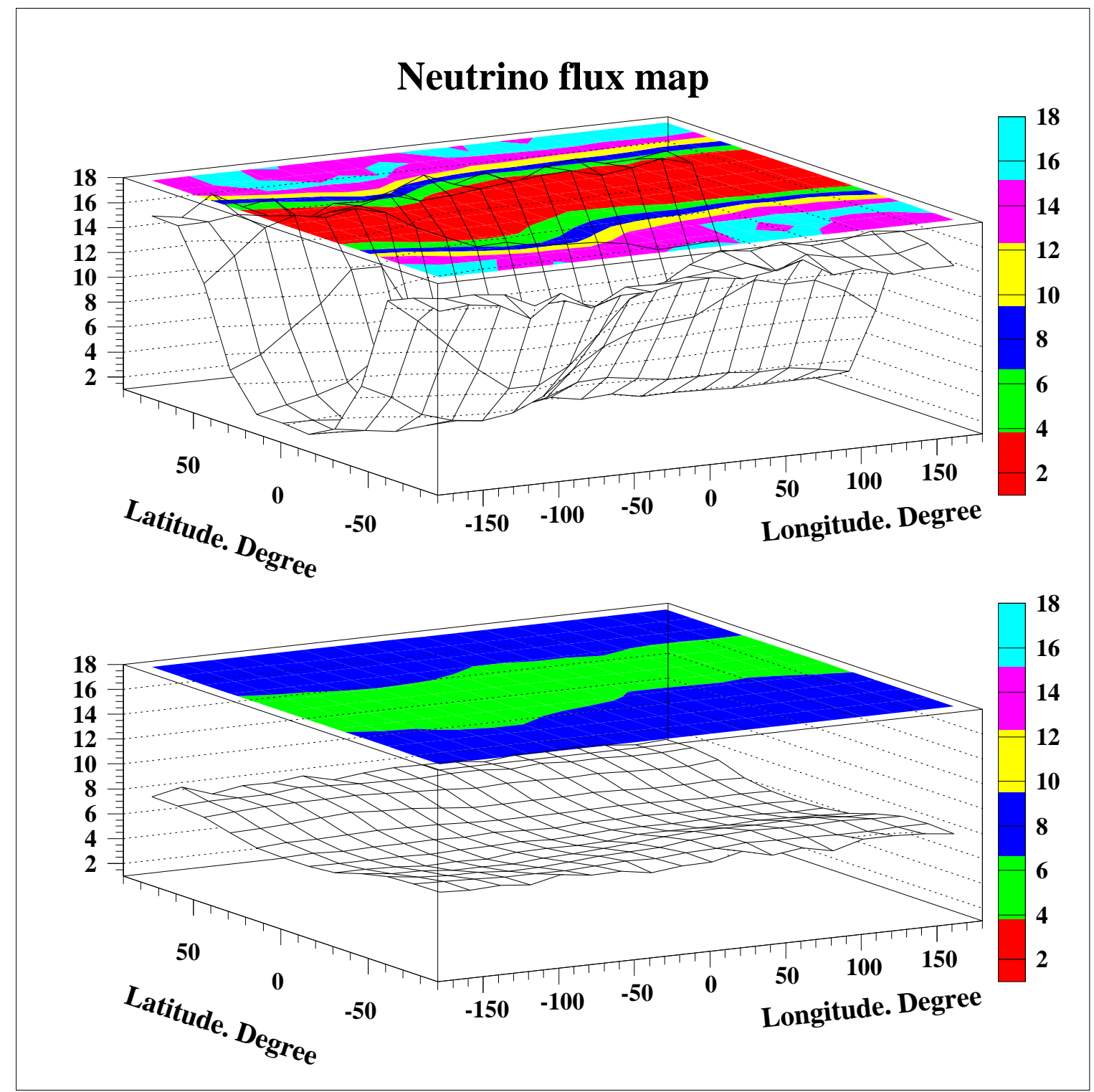

Figure 21: The geographical map of the average atmospheric neutrino flux in $\mathrm{KHz} / \mathrm{m}^{2} \mathrm{sr}$. Top: Downward doing. Bottom:Upward going. 
The actual picture of neutrino flux distribution is very different from the average one. Fig 21 shows the geographic maps of neutrino fluxes measured in $\mathrm{KHz} / \mathrm{m}^{2} \mathrm{sr}$ for downgoing (top) and upgoing (bottom) atmospheric neutrinos. The downward flux is very nonuniform and, not surprisingly, strongly correlated with the flux of cosmic protons (see fig 91). The downward neutrino flux changes from more than $15 \mathrm{KHz} / \mathrm{m}^{2} \mathrm{sr}$ at the magnetic poles down to less than $3 \mathrm{KHz} / \mathrm{m}^{2}$ sr at the magnetic equator whereas the upward flux is almost uniform at the level of $6-7 \mathrm{KHz} / \mathrm{m}^{2} \mathrm{sr}$ over the entire Earth's surface. For different regions of the Earth not only the total flux of neutrinos is different but also the kinematical parameters of the neutrinos.

To allow the comparison with previous calculations of Ref. 15] we compute the neutrino event rate in three equal area regions according to the geomagnetic latitude, although in view of neutrino flux rapidly changing with magnetic latitude (see Fig 21) such division appears to be rather crude.

As in [15] to compute the event rates the $\nu$ fluxes have been convoluted with the neutrino cross section [41, 42], 43]. The angle in the distributions (Figs 22 23 24 25 26 27) is the neutrino one with no experimental smearing or inefficiency included. Apparently, the results of the present study differ from previous calculations both in the energy spectra and the angular distributions of atmospheric neutrinos. The flux of sub-GeV neutrino predicted by this calculation depends much stronger on the geographical region following the intensity of the primary cosmic particle flux (Fig 9). The down/up asymmetry of the flux is much stronger. There is an important increase in the horizontal flux emerging from the Earth. This flux is connected with neutrinos produced under the Earth's surface at a depth of several meters. A part of these neutrinos can be detected in the underground detectors. The actual amount of "underground" neutrinos detected in a given detector depends on the detector position depth.

\section{Atmospheric neutrino fluxes at major experimen- tal sites}

For the relatively small area corresponding to different experimental sites the statistics of the present study is high enough to make reliable conclusions for neutrinos with energies below $\sim 1.5 \mathrm{GeV}$. The parameters describing the neutrino flux at the Kamioka site are calculated for an area within the geographical coordinates $35^{\circ} \pm 12^{\circ} \mathrm{N}, 142^{\circ} \pm 12^{\circ} \mathrm{E}$ for an underground detector at a depth of $520 \mathrm{~m}$ (2700 mwe). The averaged over all directions differential energy spectra of different neutrinos and antineutrinos are given in Fig 28 together with the results from other authors. The present study gives lower neutrino rates at higher energies. The fluxes of all kinds of neutrino are strongly down/up asymmetric with a considerable contribution of "underground" neutrinos in the horizontal direction (Fig 29] $)$. The ratio of $\operatorname{muon}\left(\nu_{\mu}+\tilde{\nu}_{\mu}\right)$ to electron $\left(\nu_{e}+\tilde{\nu}_{e}\right)$ fluxes (Fig 29k) suggests a tendency to decrease for horizontal directions. 


\section{Electron events. Equator region}
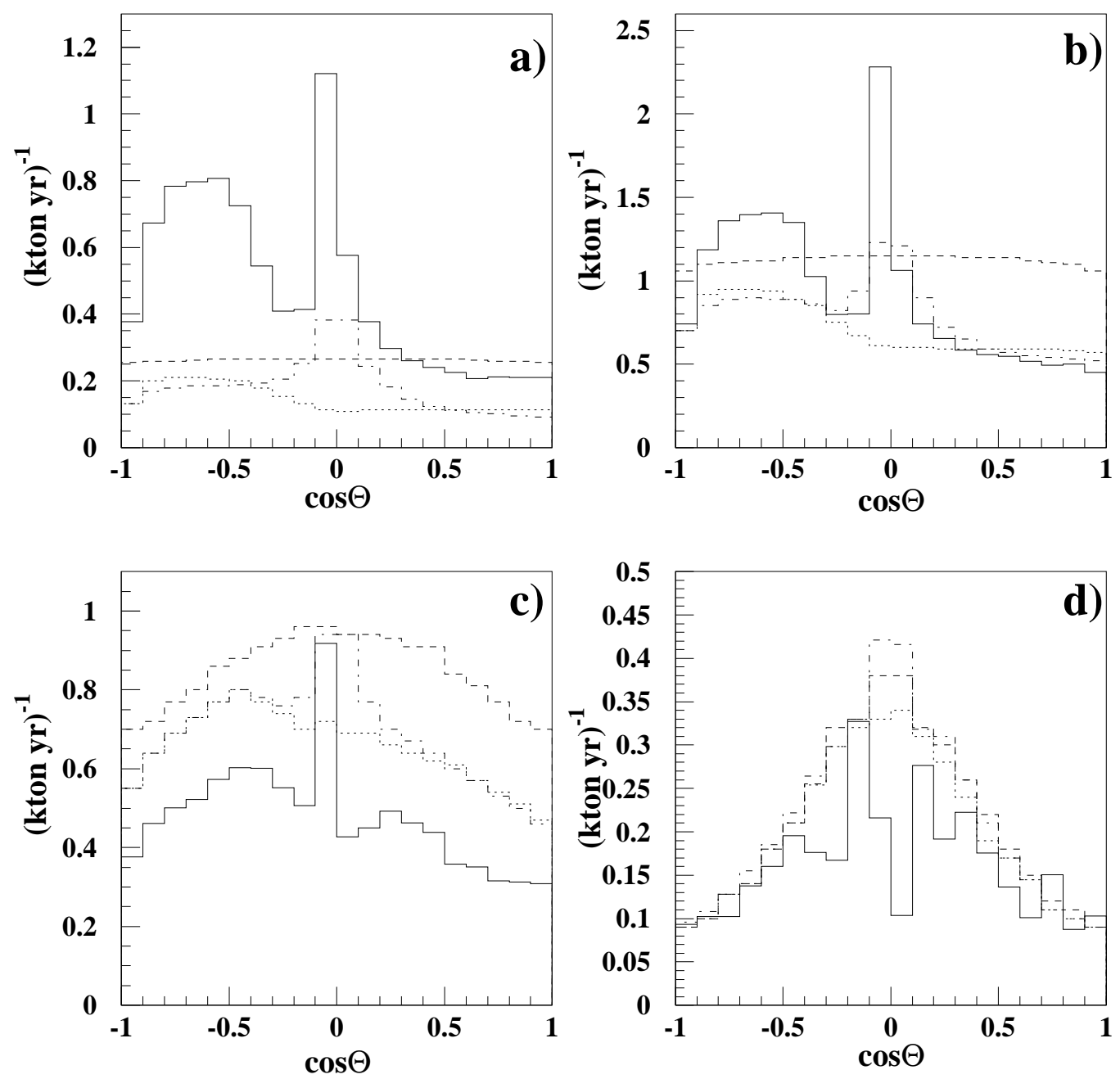

Figure 22: Average nadir angle distribution for e-like events for detectors located in positions on the Earth with magnetic latitude $\sin \left(\Theta_{\mathrm{M}}\right)=[-0.2,0.2]$. a) $\mathrm{E}_{\nu}=0.1-0.31 \mathrm{GeV}$, b) $\mathrm{E}_{\nu}=0.31-1.0 \mathrm{GeV}$, c) $\mathrm{E}_{\nu}=1.0-3.1 \mathrm{GeV}$, d) $\mathrm{E}_{\nu}=3.1-10.0 \mathrm{GeV}$. From Ref. 15 : dashed line - 1D calculation without geomagnetic effects; dotted line - 1D calculation; dash-dotted line - 3D calculation. Solid line -this work. 


\section{Electron events. Intermediate region}
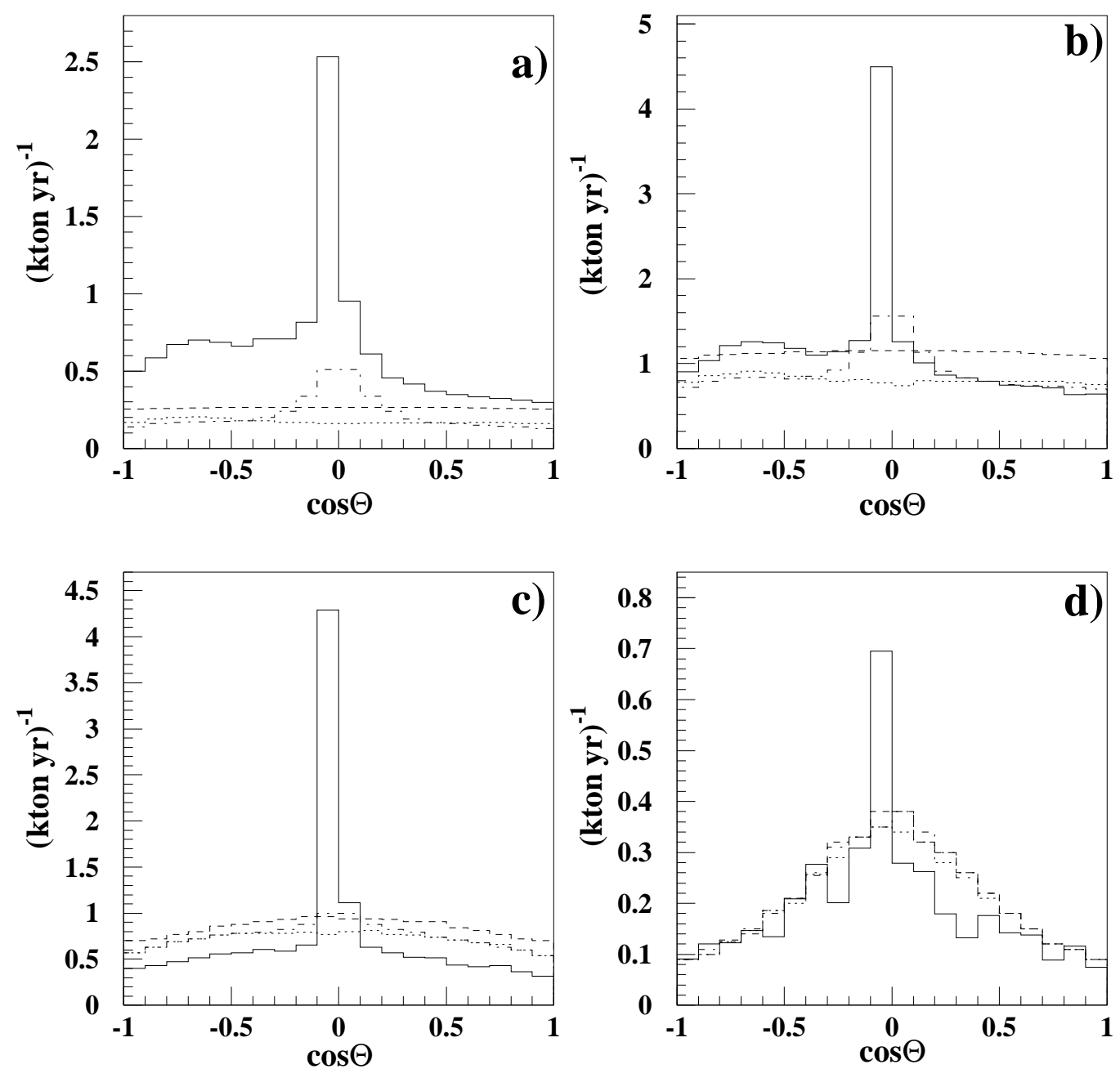

Figure 23: Average nadir angle distribution for e-like events for detectors located in positions on the Earth with magnetic latitude $\sin \left(\Theta_{\mathrm{M}}\right)=[0.2,0.6]$. a) $\mathrm{E}_{\nu}=0.1-0.31 \mathrm{GeV}$, b) $\mathrm{E}_{\nu}=0.31-1.0 \mathrm{GeV}$, c) $\mathrm{E}_{\nu}=1.0-3.1 \mathrm{GeV}$, d) $\mathrm{E}_{\nu}=3.1-10.0 \mathrm{GeV}$. From Ref. 15 : dashed line - 1D calculation without geomagnetic effects; dotted line - 1D calculation; dash-dotted line - 3D calculation. Solid line -this work. 


\section{Electron events. Polar region}
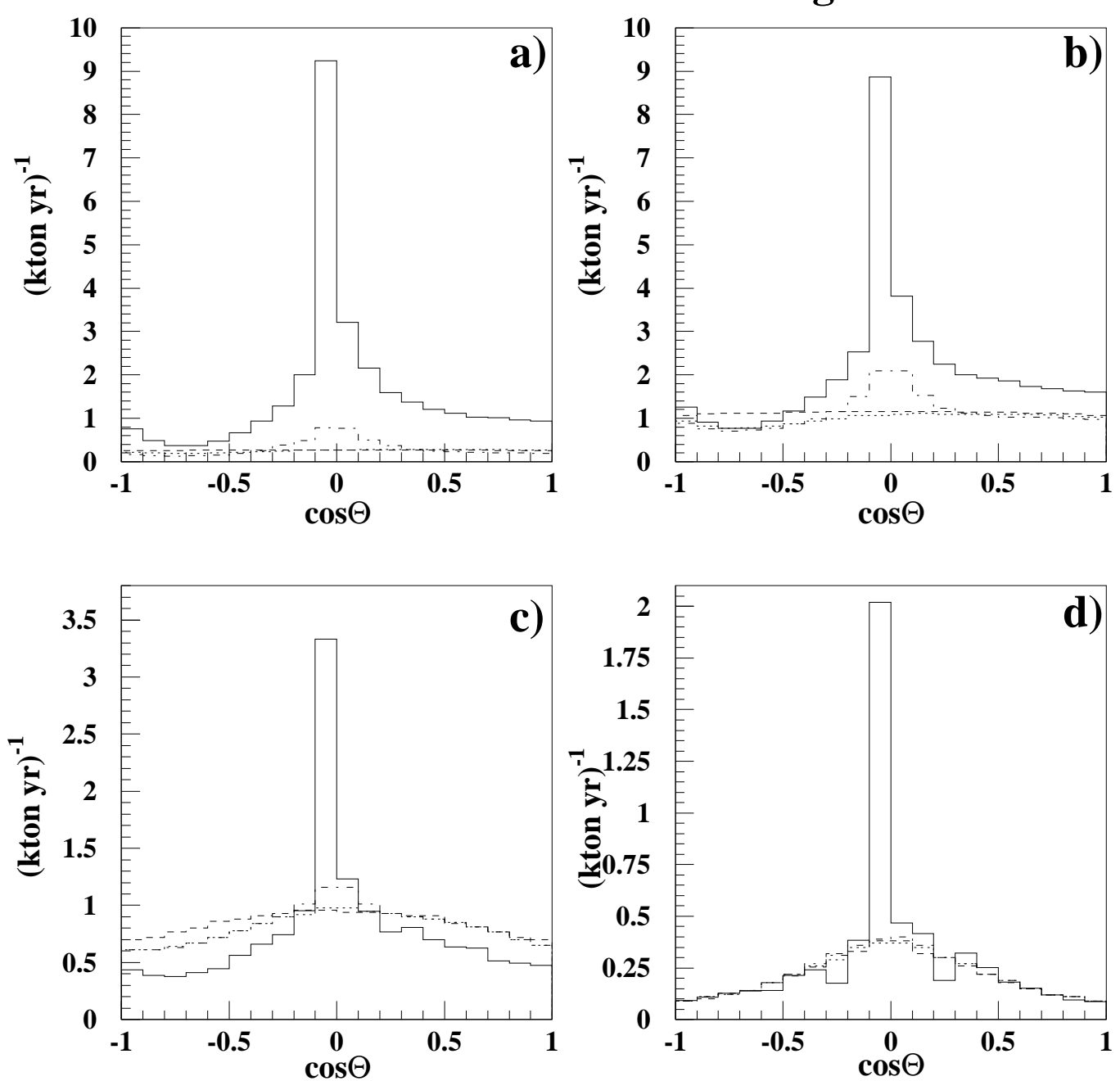

Figure 24: Average nadir angle distribution for e-like events for detectors located in positions on the Earth with magnetic latitude $\sin \left(\Theta_{\mathrm{M}}\right)=[0.6,1.0]$. a) $\mathrm{E}_{\nu}=0.1-0.31 \mathrm{GeV}$, b) $\mathrm{E}_{\nu}=0.31-1.0 \mathrm{GeV}$, c) $\mathrm{E}_{\nu}=1.0-3.1 \mathrm{GeV}$, d) $\mathrm{E}_{\nu}=3.1-10.0 \mathrm{GeV}$. From Ref. 15 : dashed line - 1D calculation without geomagnetic effects; dotted line - 1D calculation; dash-dotted line - 3D calculation. Solid line -this work. 


\section{Muon events. Equator region}
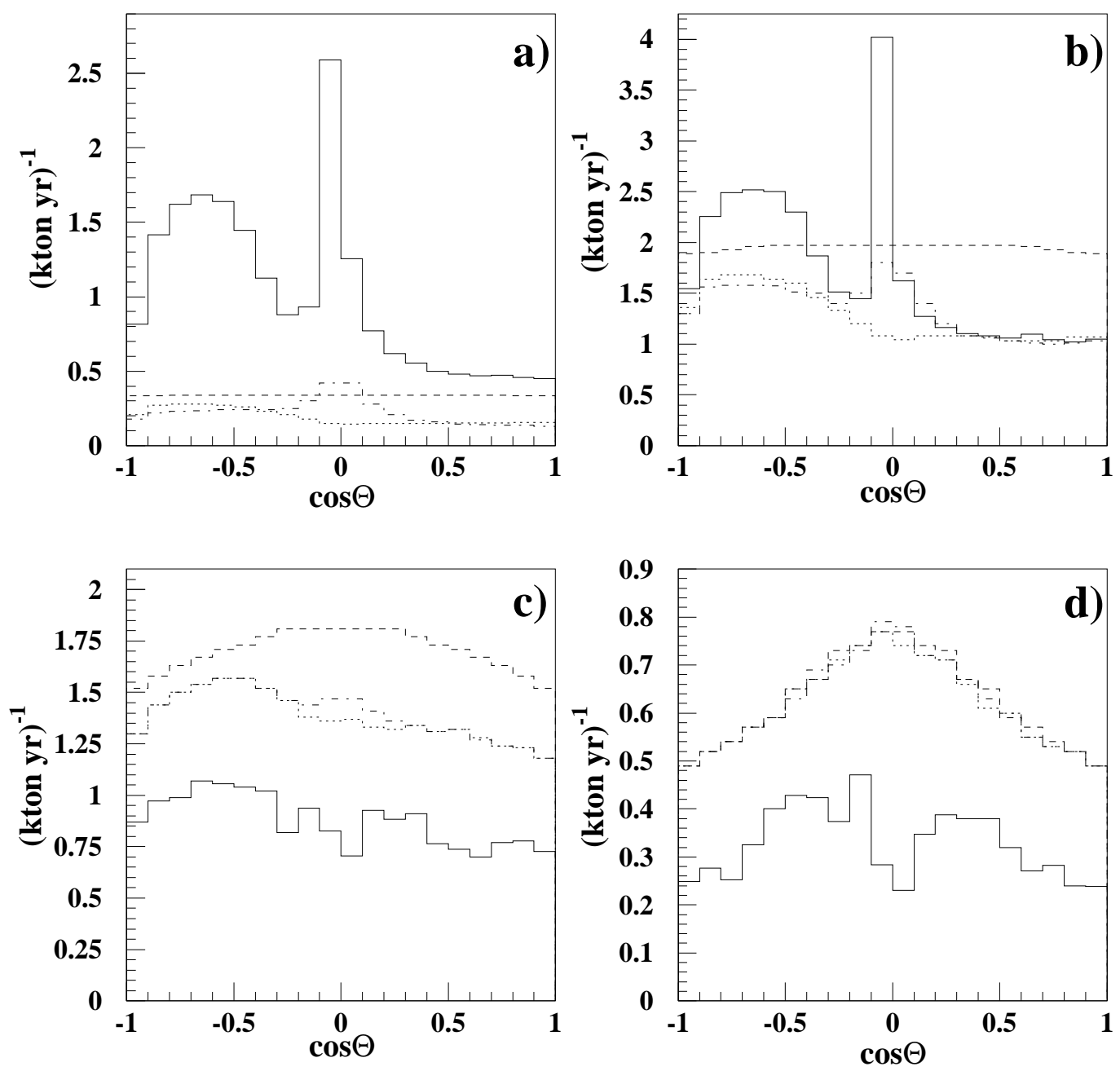

Figure 25: Average nadir angle distribution for $\mu$-like events for detectors located in positions on the Earth with magnetic latitude $\sin \left(\Theta_{\mathrm{M}}\right)=[-0.2,0.2]$. a) $\mathrm{E}_{\nu}=0.1-0.31 \mathrm{GeV}$, b) $\mathrm{E}_{\nu}=0.31-1.0 \mathrm{GeV}$, c) $\mathrm{E}_{\nu}=1.0-3.1 \mathrm{GeV}$, d) $\mathrm{E}_{\nu}=3.1-10.0 \mathrm{GeV}$. From Ref. 15 : dashed line - 1D calculation without geomagnetic effects; dotted line - 1D calculation; dash-dotted line - 3D calculation. Solid line -this work. 


\section{Muon events. Intermediate region}
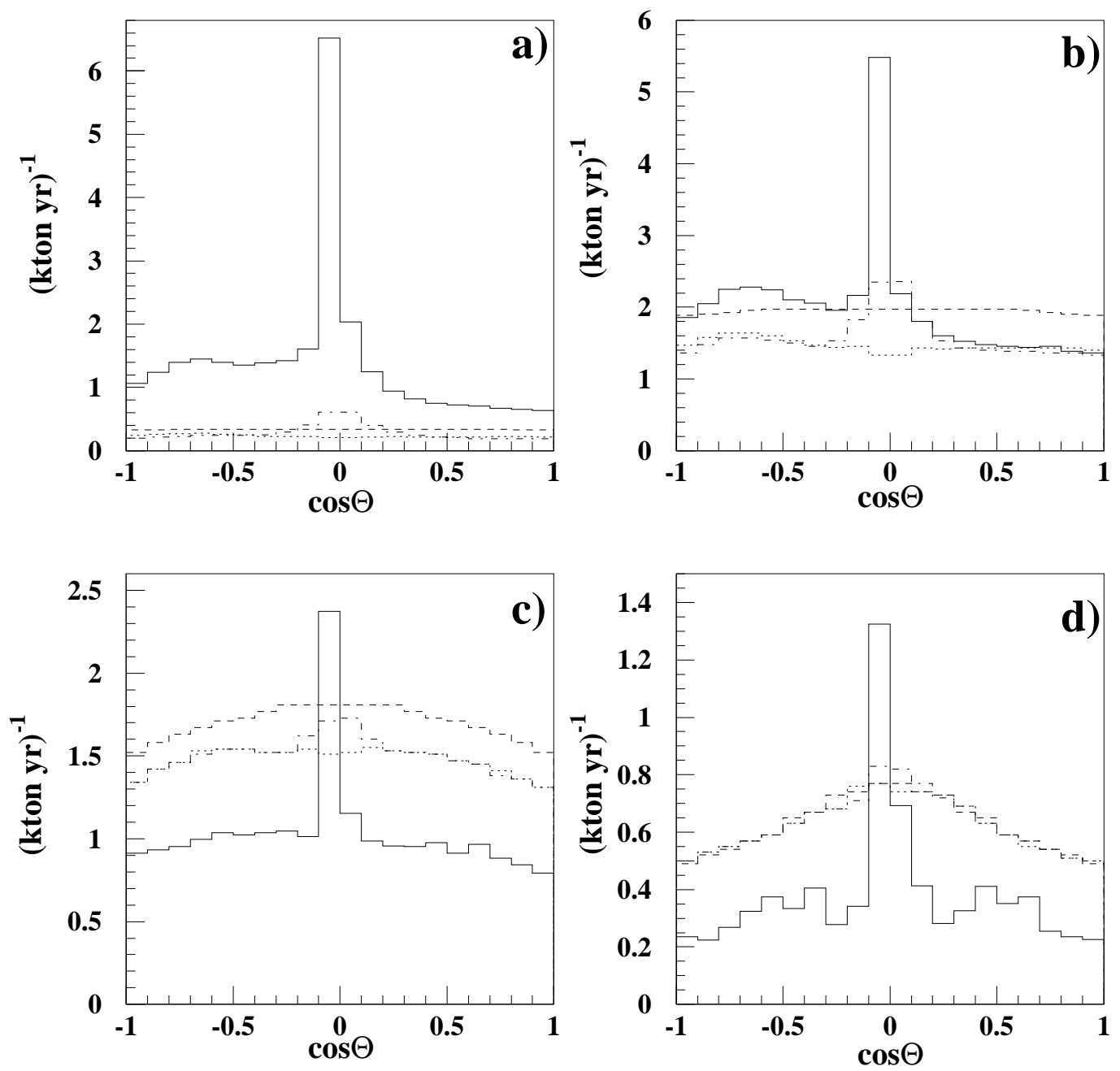

Figure 26: Average nadir angle distribution for $\mu$-like events for detectors located in positions on the Earth with magnetic latitude $\sin \left(\Theta_{\mathrm{M}}\right)=[0.2,0.6]$. a) $\mathrm{E}_{\nu}=0.1-0.31 \mathrm{GeV}$, b) $\mathrm{E}_{\nu}=0.31-1.0 \mathrm{GeV}$, c) $\mathrm{E}_{\nu}=1.0-3.1 \mathrm{GeV}$, d) $\mathrm{E}_{\nu}=3.1-10.0 \mathrm{GeV}$. From Ref. 15 : dashed line - 1D calculation without geomagnetic effects; dotted line - 1D calculation; dash-dotted line - 3D calculation. Solid line -this work. 
Muon events. Polar region
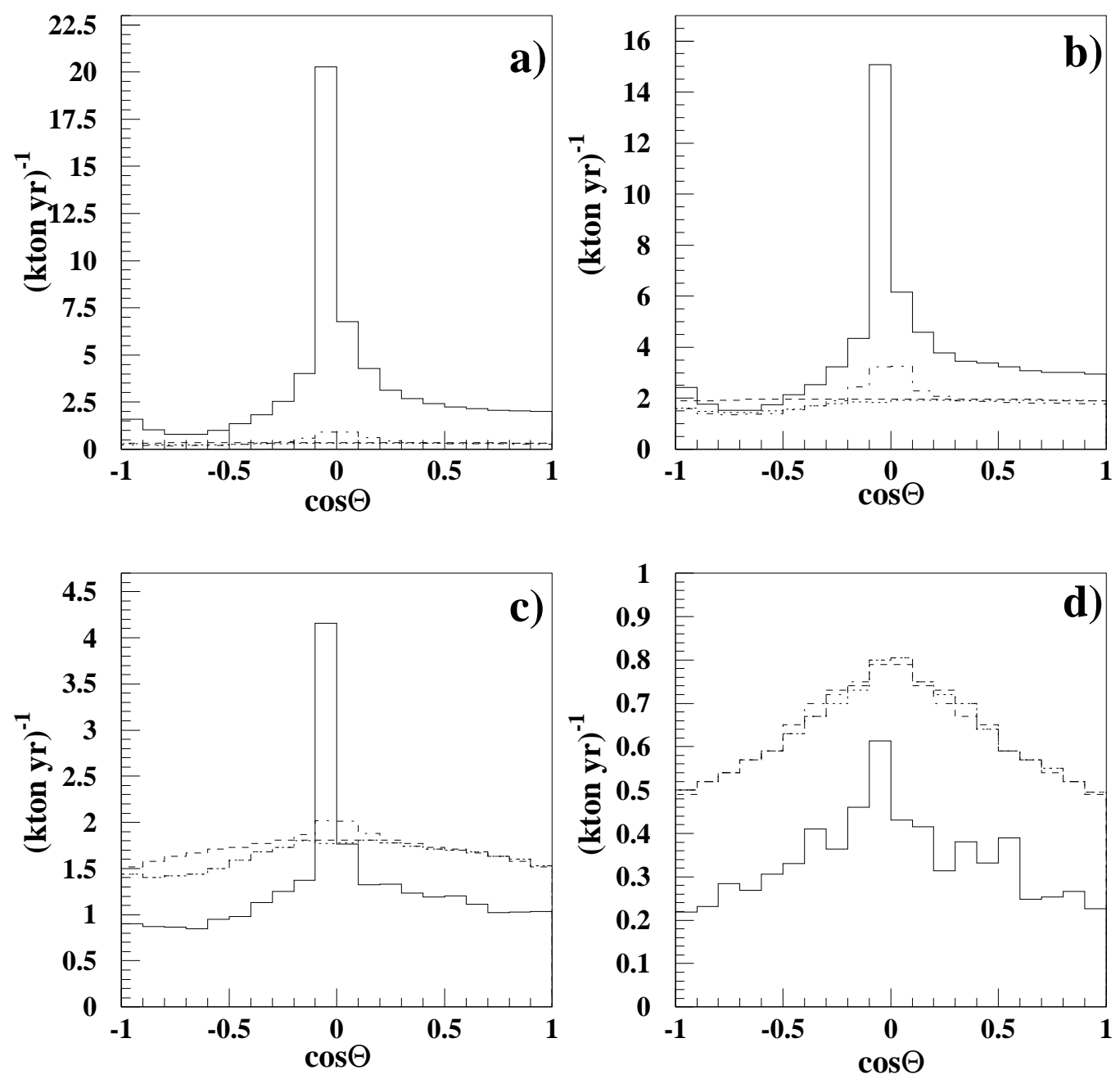

Figure 27: Average nadir angle distribution for $\mu$-like events for detectors located in positions on the Earth with magnetic latitude $\sin \left(\Theta_{\mathrm{M}}\right)=[0.6,1.0]$. a) $\mathrm{E}_{\nu}=0.1-0.31 \mathrm{GeV}$, b) $\mathrm{E}_{\nu}=0.31-1.0 \mathrm{GeV}$, c) $\mathrm{E}_{\nu}=1.0-3.1 \mathrm{GeV}$, d) $\mathrm{E}_{\nu}=3.1-10.0 \mathrm{GeV}$. From Ref. 15 : dashed line - 1D calculation without geomagnetic effects; dotted line - 1D calculation; dash-dotted line - 3D calculation. Solid line -this work. 
Neutrinos at the Kamioka site
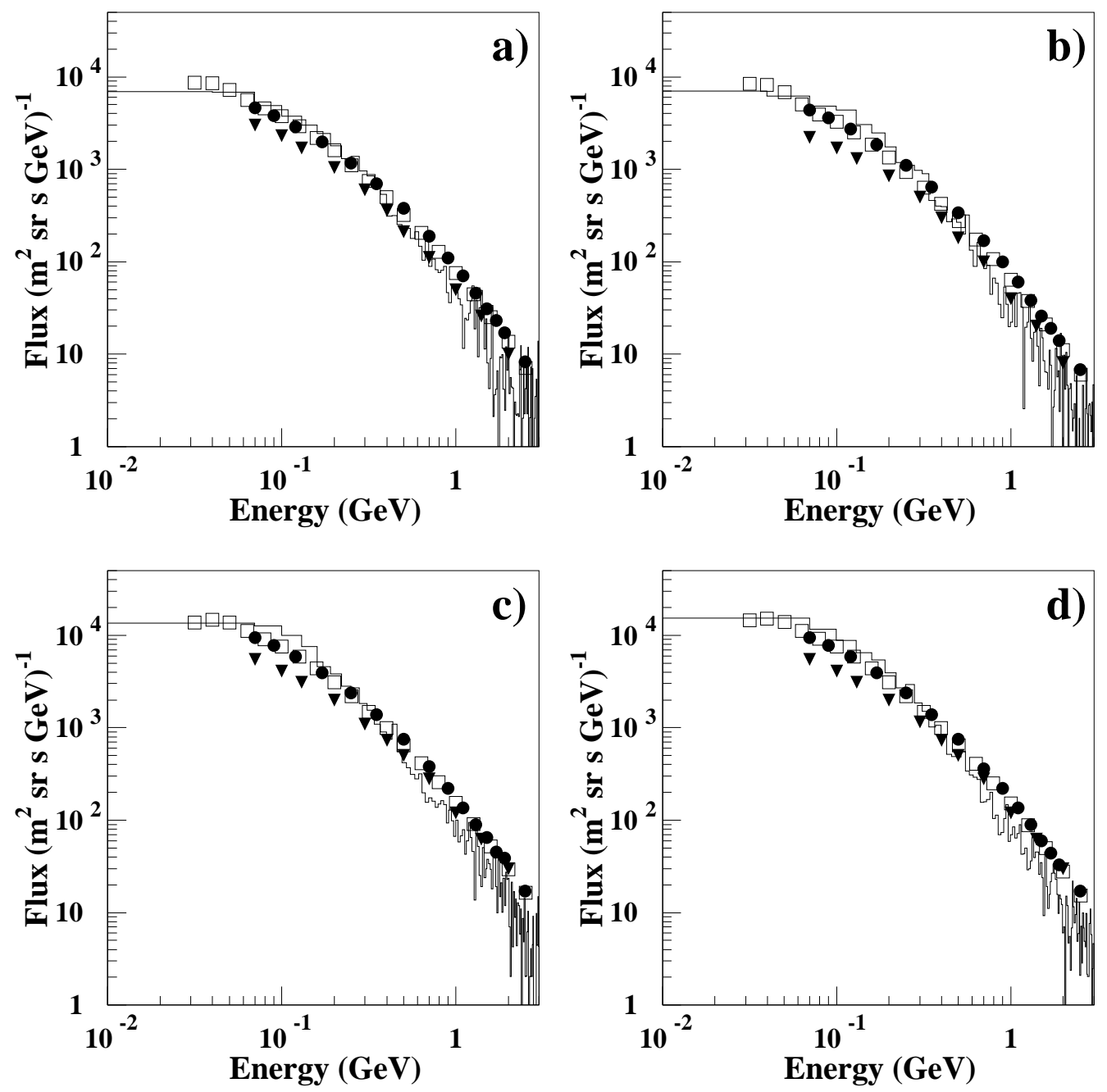

Figure 28: Differential energy spectra of atmospheric neutrino (averaged over all directions) at the Kamioka site. a) $\nu_{e}$, b) $\tilde{\nu}_{e}$, c) $\nu_{\mu}$, d) $\tilde{\nu}_{\mu}$. Squares from Ref. [7], dots from Ref. [8], triangles from Ref. [11]. Histograms - this work. 
Neutrinos at the Kamioka site
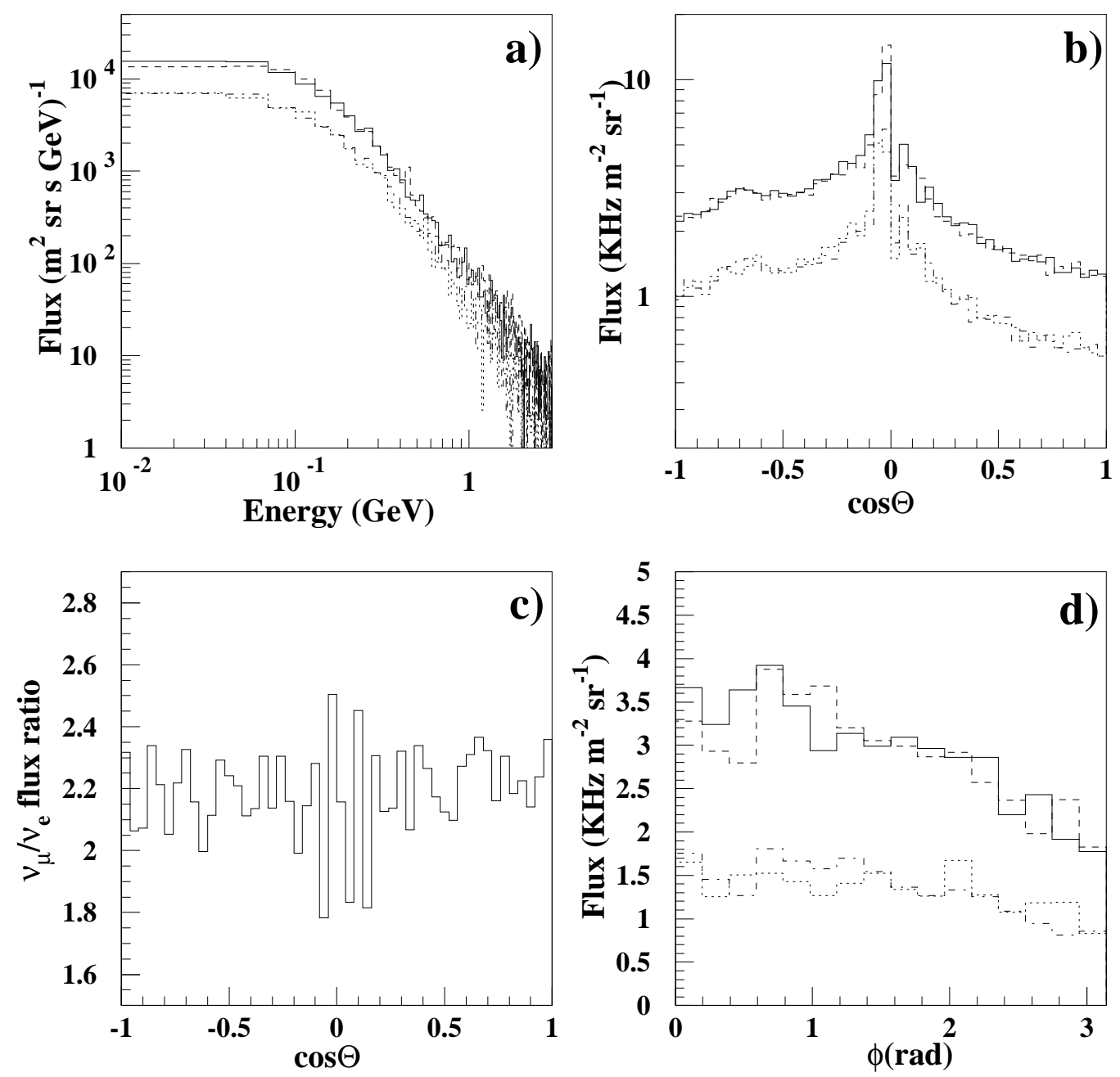

Figure 29: Neutrino flux at the Kamioka site. a) Energy spectra. b) Nadir angle distribution. c) Flux ratio of $\mu / e$ neutrinos. d) Azimuth angle distribution. In a),b) and d) solid line $-\tilde{\nu}_{\mu}$, dashed line $-\nu_{\mu}$, dotted line $-\tilde{\nu}_{e}$, dashed-dotted line $-\nu_{e}$. 
Neutrinos at the Kamioka site
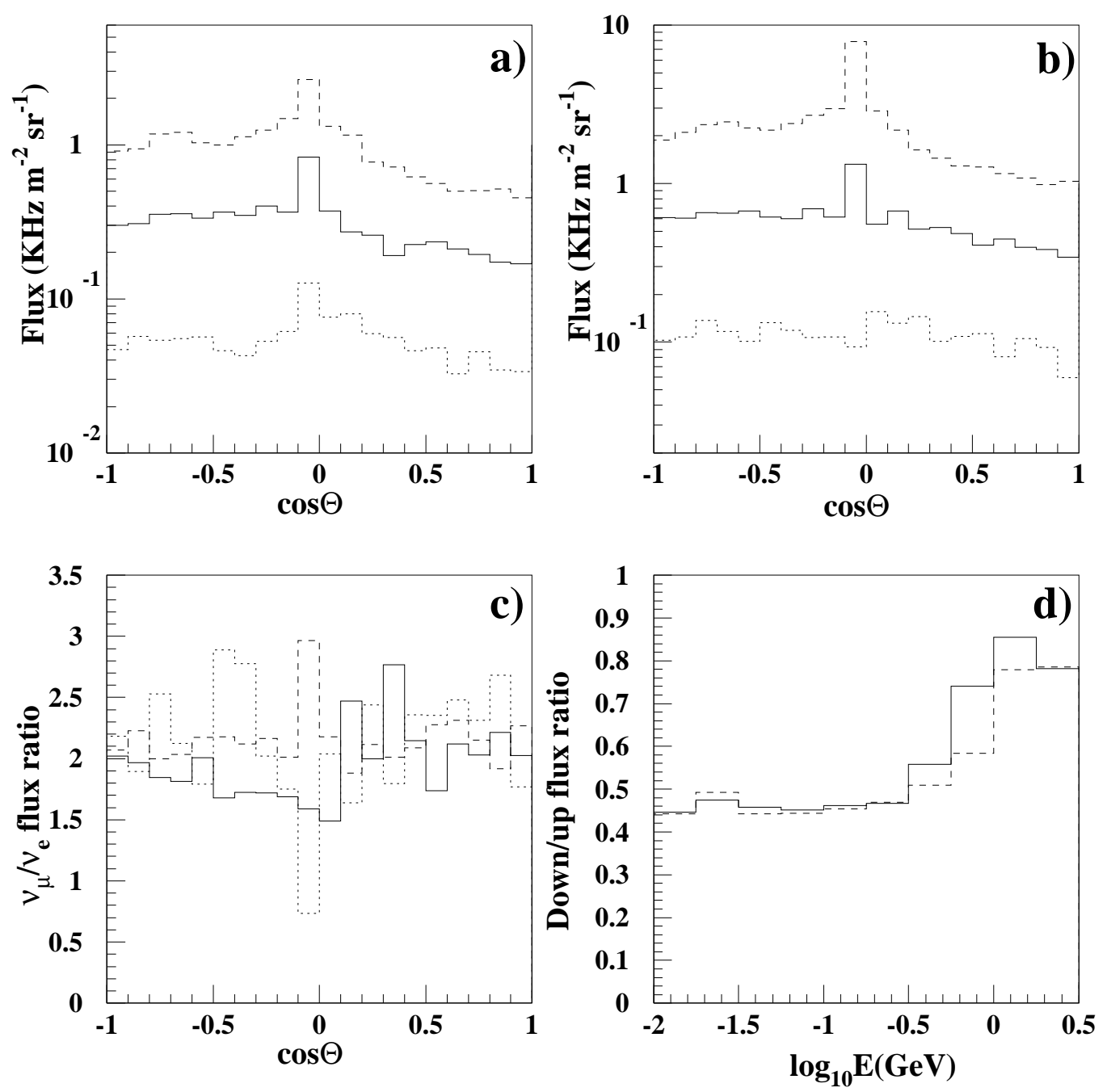

Figure 30: Neutrino flux at the Kamioka site. Nadir angle distribution for a) electron $\left(\nu_{e}, \tilde{\nu}_{e}\right)$ and b) muon $\left(\nu_{\mu}, \tilde{\nu}_{\mu}\right)$ neutrinos of different energy. d) Energy dependence of the ratio of the muon/electron fluxes. In a),b) and c) - dashed line $0.1<E<0.31 \mathrm{GeV}$, solid line $0.31<E<1.0 \mathrm{GeV}$, dotted line $E>1.0 \mathrm{GeV}$. d) Energy dependence of the flux ratio of downgoing to upgoing muon (solid line) and electron (dashed line) neutrinos. 
Neutrinos at the Soudan site
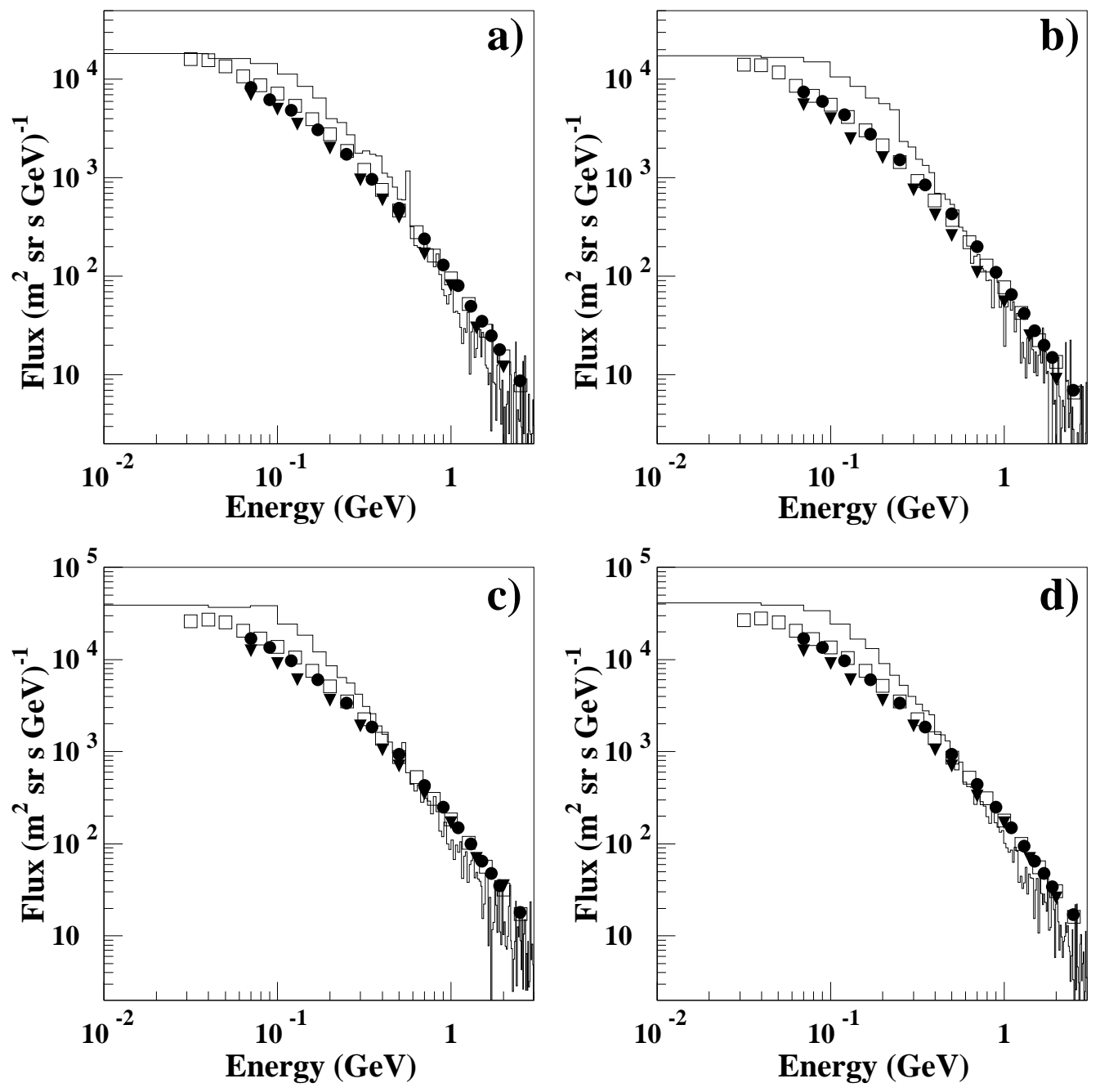

Figure 31: Differential energy spectra of atmospheric neutrino (averaged over all directions) at the Saudan site. a) $\nu_{e}$, b) $\tilde{\nu}_{e}$, c) $\nu_{\mu}$, d) $\tilde{\nu}_{\mu}$. Squares from Ref. [7], dots from Ref. 8], triangles from Ref.[11. Histograms - this work. 

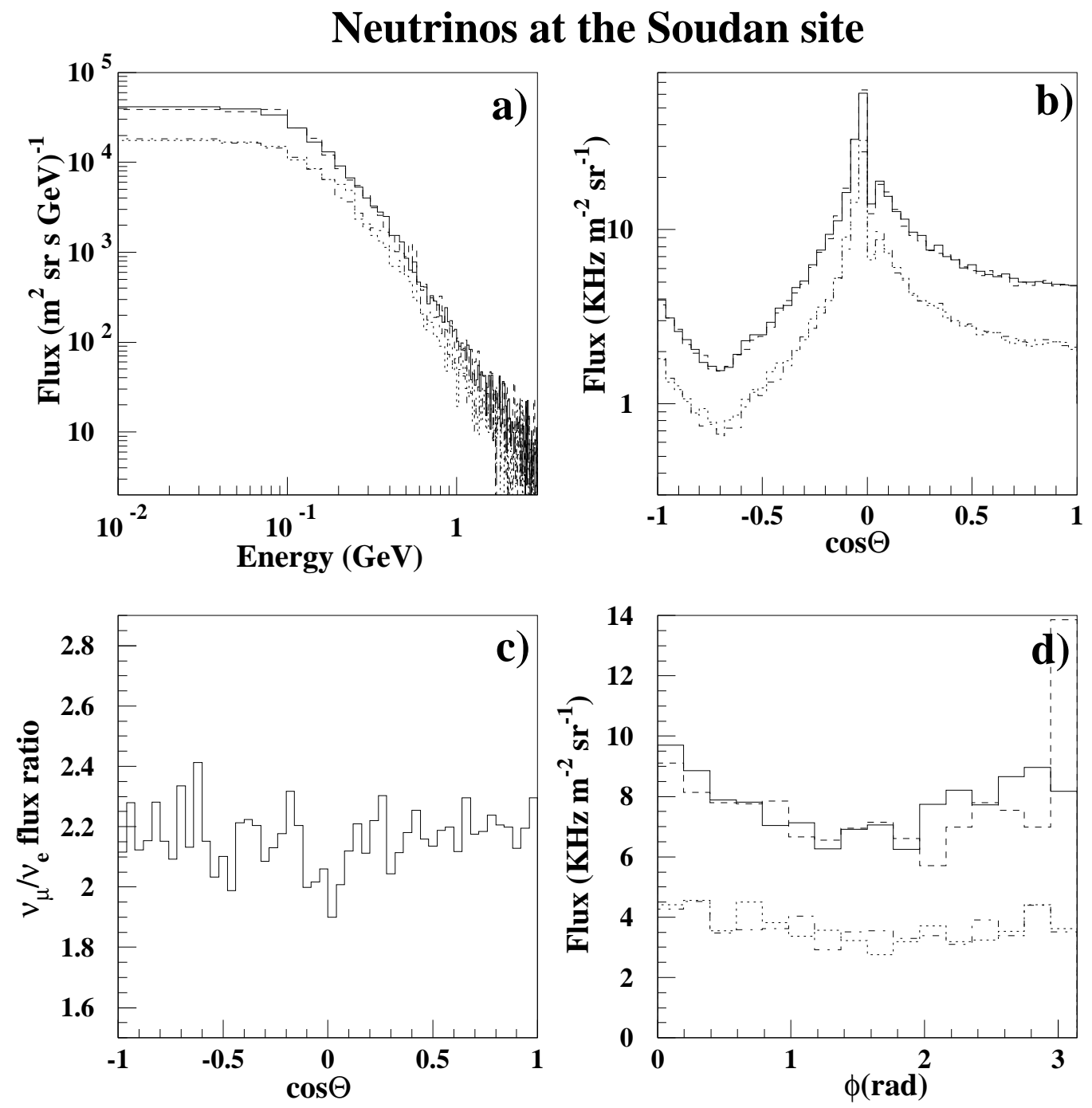

Figure 32: Neutrino flux at the Soudan site. a) Energy spectra. b) Nadir angle distribution. c)Flux ratio of $\mu / e$ neutrinos. d) Azimuth angle distribution. In a),b) and d) solid line $-\tilde{\nu}_{\mu}$, dashed line $-\nu_{\mu}$, dotted line $-\tilde{\nu}_{e}$, dashed-dotted line $-\nu_{e}$. 
Neutrinos at the Soudan site
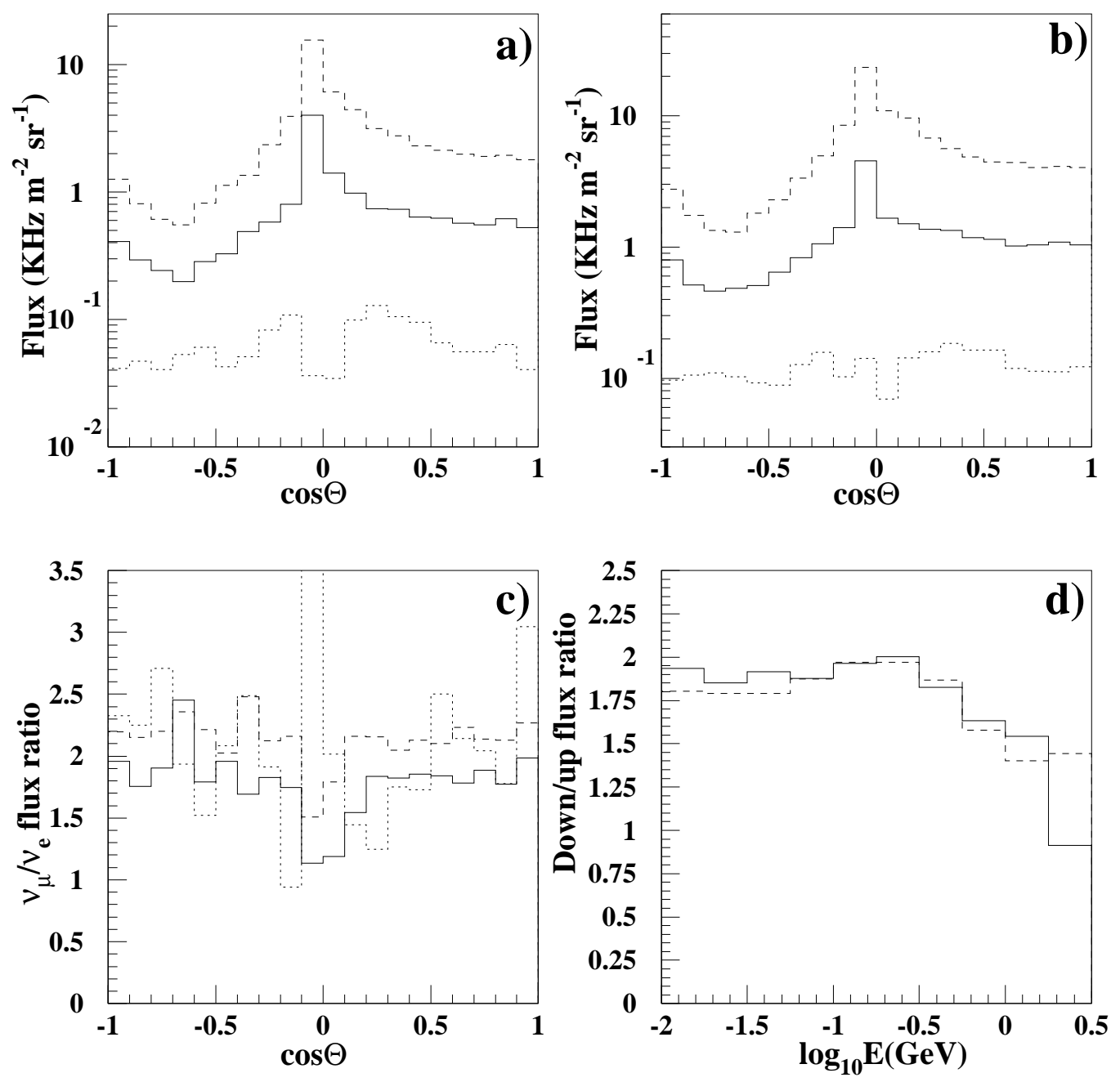

Figure 33: Neutrino flux at the Soudan site. Nadir angle distribution for a) electron $\left(\nu_{e}, \tilde{\nu}_{e}\right)$ and b) muon $\left(\nu_{\mu}, \tilde{\nu}_{\mu}\right)$ neutrinos of different energy. d) Energy dependence of the ratio of the muon/electron fluxes. In a),b) and c) - dashed line $0.1<E<0.31 \mathrm{GeV}$, solid line $0.31<E<1.0 \mathrm{GeV}$, dotted line $E>1.0 \mathrm{GeV}$. d) Energy dependence of the flux ratio of downgoing to upgoing muon (solid line) and electron (dashed line) neutrinos. 
Neutrinos at the Gran Sasso site
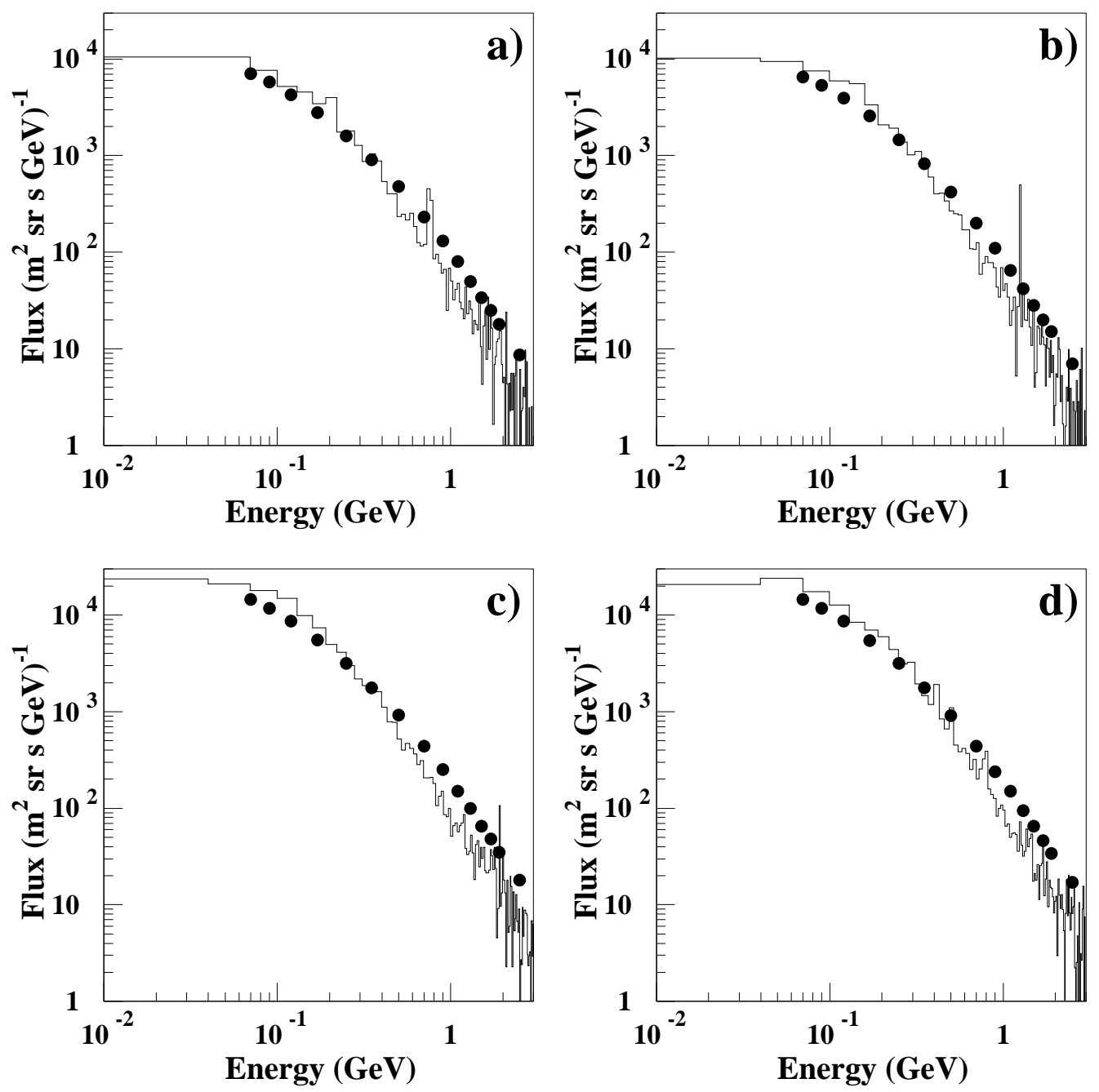

Figure 34: Differential energy spectra of atmospheric neutrino (averaged over all directions) at the Gran Sasso site. a) $\nu_{e}$, b) $\tilde{\nu}_{e}$ c) $\nu_{\mu}$, d) $\tilde{\nu}_{\mu}$. Dots from Ref. [8]. Histograms this work. 
Neutrinos at the Gran Sasso site
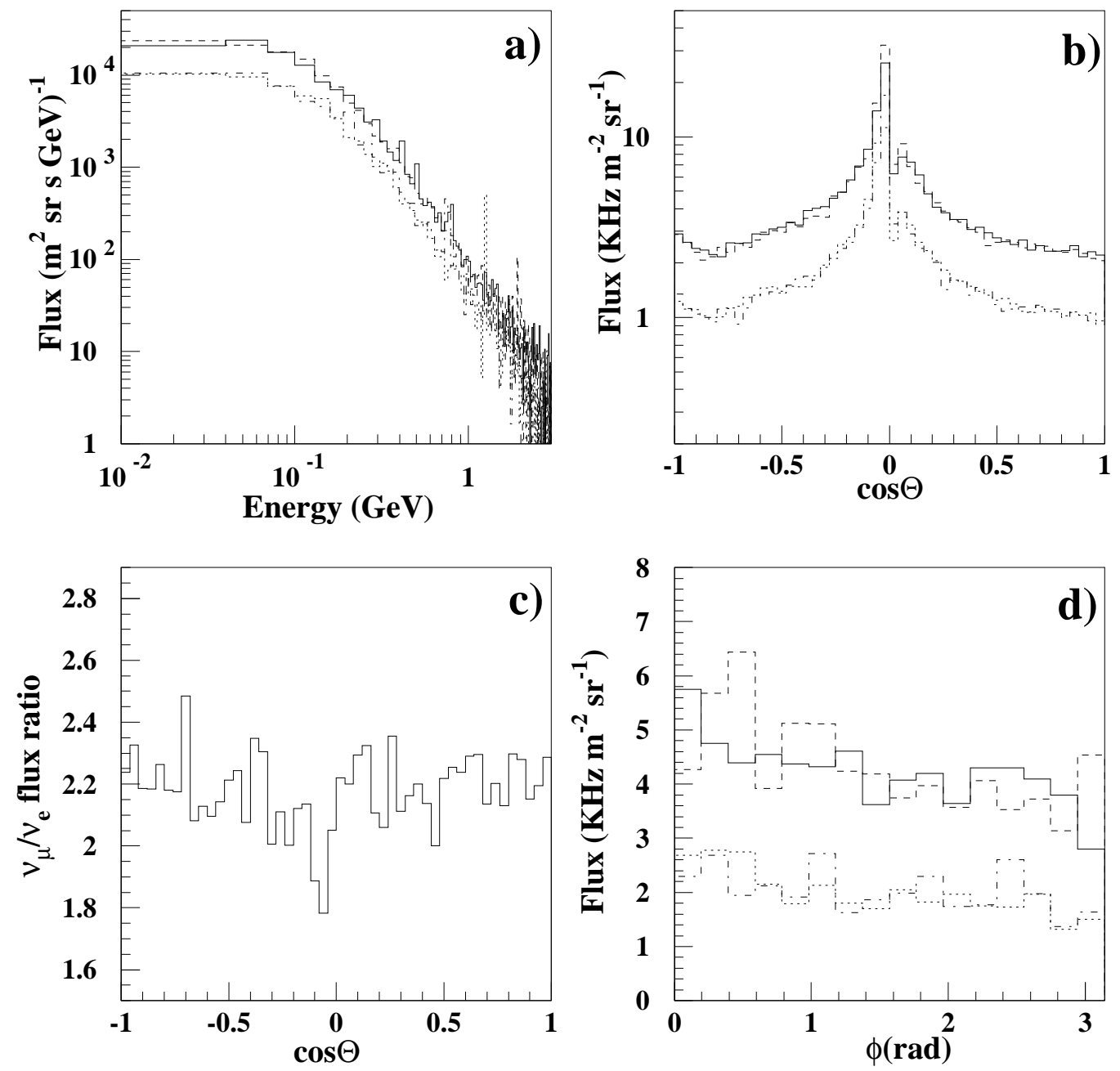

Figure 35: Neutrino flux at the Gran Sasso site. a) Energy spectra. b) Nadir angle distribution. c) Flux ratio of $\mu / e$ neutrinos. d) Azimuth angle distribution. In a),b) and d) solid line $-\tilde{\nu}_{\mu}$, dashed line $-\nu_{\mu}$, dotted line $-\tilde{\nu}_{e}$, dashed-dotted line $-\nu_{e}$. 
Neutrinos at the Gran Sasso site
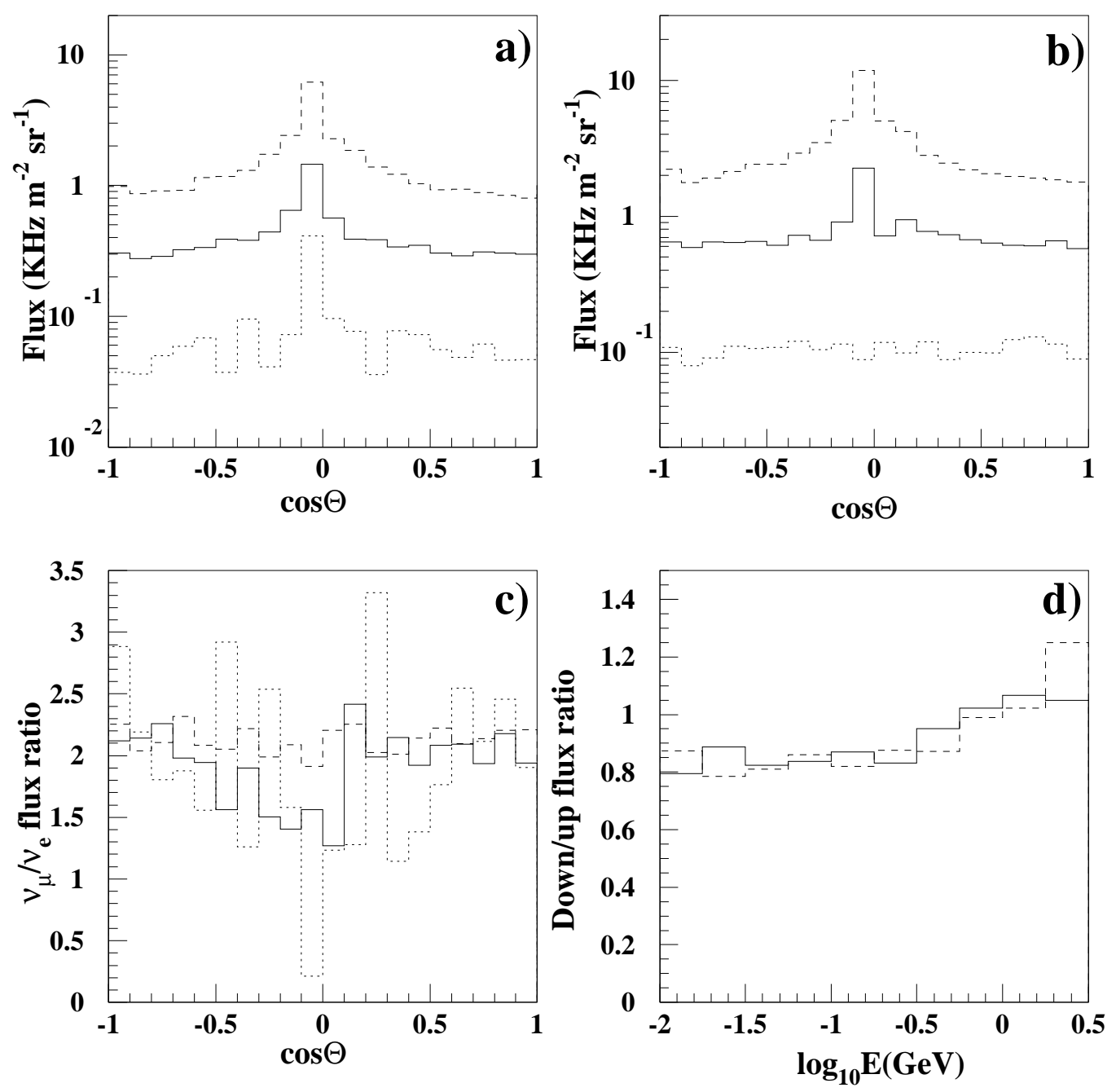

Figure 36: Neutrino flux at the Gran Sasso site. Nadir angle distribution for a) electron $\left(\nu_{e}, \tilde{\nu}_{e}\right)$ and b) muon $\left(\nu_{\mu}, \tilde{\nu}_{\mu}\right)$ neutrinos of different energy. d) Energy dependence of the ratio of the muon/electron fluxes.In a),b) and c) - dashed line $0.1<E<0.31 \mathrm{GeV}$, solid line $0.31<E<1.0 \mathrm{GeV}$, dotted line $E>1.0 \mathrm{GeV}$. d) Energy dependence of the flux ratio of downgoing to upgoing muon (solid line) and electron (dashed line) neutrinos. 
This systematic effect for neutrinos with the highest flux (see Fig 29b) if not taken into account may lead to a conclusion about $\nu_{\mu}, \tilde{\nu}_{\mu}$ disappearance. The east-west asymmetry of electron and muon neutrino is visible in Fig $29 \mathrm{~d}$.

The energy dependence of the angular distribution of the flux of electron and muon neutrino is illustrated by Figs 30 and $30 \mathrm{~b}$ correspondingly. Note the absence of the increase in the muon neutrino flux in the horizontal direction. Figure $30 \mathrm{~d}$ shows the energy dependence of the downward/upward flux ratio for muon (solid line) and electron (dashed line) neutrinos. The ratio is the same for two different kinds of neutrino in the energy range below 0.31 and above $1.5 \mathrm{GeV}$. However, for the region between 0.31 and $1.5 \mathrm{GeV}$ where the bulk of the experimental data is collected the down/up ratio for muon neutrinos is systematically higher than that for electron neutrinos. This systematic effect may fake muon neutrino disappearance.

Neutrino fluxes at the Soudan and Gran Sasso sites are calculated for the areas with geographical coordinates of $48^{\circ} \pm 12^{\circ} \mathrm{N}, 98^{\circ} \pm 12^{\circ} \mathrm{W}$ and $42^{\circ} \pm 12^{\circ} \mathrm{N}, 13^{\circ} \pm 12^{\circ} \mathrm{E}$ and the depth underground of $700 \mathrm{~m}$ and $750 \mathrm{~m}$ correspondingly. As for the Kamioka site the averaged over all directions differential energy spectra of different neutrinos and antineutrinos at the Soudan (Fig 31) and Gran Sasso (Fig 34) sites obtained in the present calculation give lower neutrino rates at higher energies than those obtained by other authors. The angular distributions of neutrino flux is strongly up/down asymmetric with predominance of the downward flux at the Soudan site (Fig 32b) whereas it is almost up/down symmetric for Gran Sasso (Fig 35b). The value and the energy dependence of the downward/upward flux ratio (Figs 33 36 d) for muon (solid line) and electron (dashed line) neutrinos is different for the two sites. At both experimental sites this ratio is almost the same for neutrinos of different kind with energies less than $1.5 \mathrm{GeV}$. There is no strong indication that the ratio of muon to electron fluxes at the Soudan site (Fig 32 33d) depends on the neutrino direction. On the contrary the tendency for the ratio of muon to electron fluxes to decrease for directions close to a horizontal plane is clearly seen for neutrinos in the energy range 0.31 - $1.0 \mathrm{GeV}$ in Gran Sasso (solid line in Fig 36r).

\section{Summary and conclusions}

The proton flux near the Earth consists of primary protons from space, primary protons scattered in the atmosphere and secondary protons produced in atmospheric showers. Each component of the proton flux has different angular distribution. The results of the above Monte Carlo study of propagation in space and interaction with the atmosphere of cosmic particles show that the primary proton flux in the vicinity of the Earth is essentially anisotropic at the directions approaching the horizontal plane. This result is in contradiction with the assumption of most atmospheric neutrino flux calculations. The anisotropy has an important impact on the production of higher energy neutrino. 
The momentum spectra of primary protons from space display at different latitudes the expected [22, 36, 37] behaviour connected with the Earth's magnetic field (cutoff). There exist, however, a non negligible amount of primary protons with momenta well below the magnetic cutoff.

The momenta of the considerable number of scattered primary protons present at all magnetic latitudes are close to the cutoff value. When backtraced, the scattered protons have "forbidden" trajectories and consequently their contribution to atmospheric neutrino production was not taken into account in previous calculations of neutrino fluxes.

Predominantly secondary under cutoff particles (protons, $e^{+}, e^{-}$) are present in equal amount and the same momenta in the fluxes of particles going to and from the Earth. The simulation correctly describes the fluxes of protons and leptons $\left(e^{+}, e^{-}\right)$measured by AMS [24, 25, 40].

The atmospheric neutrinos produced in the simulation have the energy spectra, relative fluxes and angular distributions different from those obtained in the previous atmospheric neutrino flux calculations [6, 17, 8, 9, 10, 11, 12, 14, 15]. The difference is a direct consequence of the anisotropy of the cosmic ray flux at the vicinity of the Earth. As it follows from the present study, the flux of primary cosmic particles at their entrance into the atmosphere is diminishing when approaching the horizontal direction (see Fig 11). Because of that, the fraction of high energy secondaries capable to decay before they lose most of their energy on the ionization of the atmosphere decreases. This results in smaller neutrino fluxes at higher energies.

The up/down asymmetry of the neutrino fluxes resulting from this study is essentially different from the previous calculations. Similar to previous 3-D calculations the flux of atmospheric neutrinos has a maximum in the angular region around the horizontal plane. However, as it is demonstrated in this study there is an important contribution to the horizontal flux from neutrinos produced just under the Earth's surface. A part of the flux of the "underground" neutrinos can be detected in the underground experiments. The horizontal region is the most important for determination of the up/down asymmetry of the atmospheric neutrino fluxes and consequently the result of the present study may affect the eventual experimental measurement of the neutrino oscillation parameter $\Delta \mathrm{m}^{2}$. Calculated for different experimental sites the angular distributions of neutrino fluxes of different kind and their dependence on the neutrino energy suggest the presence of effects faking disappearance of upward going muon neutrinos. These effects are different at different experimental sites (Figures 30k, d; 33k, d 36k, d). This could probably be the reason why different experiments do not agree in their estimate of oscillation parameters.

Acknowlegements. I'm grateful to Prof.Yu.Galaktionov for very useful and stimulating discussions. I have to express my gratitude to Dr.V.Shoutko for reading the manuscript and giving suggestions for important improvements. Finaly I would like to thank Prof.S.C.C.Ting for his interest in this work and encouragement. 


\section{References}

[1] Super-Kamiokande collaboration, Y.Fukuda et al, Phys. Rev. Lett. 81, 1562 (1998).

[2] Kamiokande collaboration, K.S.Hirata et al, Phys. Lett. B 205, 416 (1988); Phys. Lett. B 289, 146 (1992); Y.Fukuda et al, Phys. Lett. B 335, 237 (1994).

[3] IMB collaboration, D.Casper et al, Phys. Rev. Lett. 66, 2561 (1991); R.Becker-Szendy et al, Phys. Rev. D 46, 3720 (1992).

[4] Soudan collaboration, W.W.M.Allison et al, Phys. Lett. B 434, 137 (1999).

[5] MACRO collaboration, Phys. Lett. B 434, 451 (1998); Preprint hep-ex/0001044 submitted to Phys. Lett. B (2000).

[6] M.Honda et al, Phys. Lett. B 248, 193 (1990).

[7] M.Honda et al, Phys. Rev. D 52, 4985 (1995).

[8] G.Barr,T.K.Gaisser and T.Stanev, Phys. Rev. D 39, 3532 (1989).

[9] G.Barr,T.K.Gaisser and T.Stanev,Phys. Rev. D 38, 85 (1989); V.Agraval et al, Phys. Rev. D 53, 1314 (1996).

[10] L.V.Volkova, Yad.Fiz. 31, 1510 (1980) also in Sov.J.Nucl.Phys. 31, 784 (1980).

[11] E.V.Bugaev and V.A.Naumov, Phys. Lett. B 232, 391 (1989).

[12] A.V.Butkevich, L.G.Dedenko and I.M.Zheleznykh, Yad.Fiz. 50, 142 (1989) also in Sov.J.Nucl.Phys. 50, 90 (1989).

[13] P.Lipari, Astroparticle Physics 1, 195 (1993).

[14] G.Battistoni et al, Astroparticle Physics 12, 315 (2000).

[15] P.Lipari, Astroparticle Physics 14, 153 (2000).

[16] Super-Kamiokande collaboration, T.Futagami et al, Phys. Rev. Lett. 82, 5194 (1999).

[17] P.Lipari,T.K.Gaisser and T.Stanev, Phys. Rev. D 58, 3003 (1998).

[18] T.H.Johnson, Phys. Rev.43, 307 (1933); Phys. Rev.43, 381 (1933); Phys. Rev.48, 287 (1935).

[19] L.W.Alwarez and A.H.Compton, Phys. Rev.43, 835 (1933);

[20] P.Lipari, Preprint arXiv:hep-ph/0003013 (2000).

[21] H.R.Schmidt,J.Schukraft, J.Phys. G 19, 1705 (1993). 
[22] C.Störmer, Astrophysics 1, 237 (1933).

[23] S.Ahlen et al, NIM A 350, 351 (1994).

[24] AMS collaboration, Phys. Lett. B 472, 215 (2000).

[25] AMS collaboration, Phys. Lett. B 484, 10 (2000).

[26] GEANT 3, CERN DD/EE/84-1, Revised, 1987

[27] US Standard Atmosphere. see e.g. S.E.Forsythe, Smithsonian phusical tables.(Smithsonian Institution press.Washington,DC, 1969)

[28] S.MacMillan et al, Journal of Geomagnetism and Geoelectricity, 49, 229 (1997); J.M.Quinn et al, Journal of Geomagnetism and Geoelectricity, 49, 245 (1997);

[29] P.L.Biermann et al, Phys. Rev. D 51, 3450 (1995).

[30] I.V.Moskalenko and A.W.Strong, ApJ. 493, 694 (1998).

[31] V.L.Ginzburg and V.S.Ptushkin, Rev.Mod.Phys. 48, 171 (1976).

[32] W.R.Webber et al, ApJ. 390, 96 (1991).

[33] A.D.Erlykin and A.W.Wolfendale, Eur.J.Phys. 20, 409 (1999).

[34] W.R.Webber and. M.S.Potgieter, ApJ. 344, 779 (1989).

[35] B.Jakobsson et al, Phys. Rev. Lett. 78, 3828 (1997).

[36] C.Störmer, The Polar Aurora, Clarendon Press (Cambridge), 1955.

[37] S.F.Singer and A.M.Lenchek, Prog.in Elem.Part and Cosm. Ray Phys. Vol 6, p245, NHPC, 1962.

[38] S.C.C.Ting, in The First Arctic Workshop on Future Physics and Accelerators, ed. K.Huitu, M.Chaian and R.Orova, (World Sceintific, 1995) pp. 1-26.

[39] AMS-collaboration. "Cosmic Ray Studies by AMS precursor flight" (in preparation).

[40] V.Plyaskin,"Particles below geomagnetic cutoff", AMS internal report. February 1999. (unpublished).

[41] P.Lipari,M.Lusignoli and F.Sartogo. Phys.Rev.Lett. 74,4384 (1995).

[42] O.Erriquez et al. Phys. Lett. 80B, No.3, 309 (1979).

[43] PDB, Phys. Rev. D. 45, No.11,III.82 (1992) and references therein.

[44] O.C.Allkofer et al. Phys. Lett. B36, 425 (1971).

[45] B.C.Rastin,J.Phys. G10, 1609 (1984). 